\title{
MATHEMATICAL AND NUMERICAL ANALYSIS OF A STRATIGRAPHIC MODEL
}

\author{
VÉRonique Gervais ${ }^{1}$ AND Roland MASSON ${ }^{1}$
}

\begin{abstract}
In this paper, we consider a multi-lithology diffusion model used in stratigraphic modelling to simulate large scale transport processes of sediments described as a mixture of $L$ lithologies. This model is a simplified one for which the surficial fluxes are proportional to the slope of the topography and to a lithology fraction with unitary diffusion coefficients. The main unknowns of the system are the sediment thickness $h$, the $L$ surface concentrations $c_{i}^{s}$ in lithology $i$ of the sediments at the top of the basin, and the $L$ concentrations $c_{i}$ in lithology $i$ of the sediments inside the basin. For this simplified model, the sediment thickness decouples from the other unknowns and satisfies a linear parabolic equation. The remaining equations account for the mass conservation of the lithologies, and couple, for each lithology, a first order linear equation for $c_{i}^{s}$ with a linear advection equation for $c_{i}$ for which $c_{i}^{s}$ appears as an input boundary condition. For this coupled system, a weak formulation is introduced which is shown to have a unique solution. An implicit finite volume scheme is derived for which we show stability estimates and the convergence to the weak solution of the problem.
\end{abstract}

Mathematics Subject Classification. 35M10, 35L50, 35Q99, 65M12.

Received: February 3, 2004.

\section{INTRODUCTION}

Recent progress in geosciences, and more especially in seismic- and sequence-stratigraphy, have improved the understanding of sedimentary basins infill. Indeed, the sediments architecture is the response to complex interactions between the available space created in the basin by sea level variations, tectonic, compaction, ...., the sediment supply (boundary fluxes, sediment production), and the transport of the sediments at the surface of the basin. In order to have a quantified view of this response and to determine the relative influence of each involved process, stratigraphic models have been developed.

Among basin infill models considering the dynamics of sediment transport, authors usually distinguish between fluid-flow and dynamic-slope models (see $[12,13]$ ). The first ones use fluid flow equations and empirical algorithms to simulate the transport of sediments in the hydrodynamic flow field (see e.g. [14]). They provide an accurate description of depositional processes for small scales in time and space but, at larger scale such as basin scales, they are computationally too expensive.

Keywords and phrases. Finite volume method, stratigraphic modelling, linear first order equations, convergence analysis, linear advection equation, unique weak solution, adjoint problem.

1 Institut Français du Pétrole, 1 et 4 avenue de Bois Préau, 92852 Rueil Malmaison Cedex, France.

e-mail: veronique.gervais@ifp.fr, roland.masson@ifp.fr 
Dynamic-slope models use mass conservation equations of sediments combined with diffusive transport laws. These laws do not describe each geological process in details, but average over these processes (river transport, creep, slumps, small slides, ... ). One can refer to $[1,6,8,10,12,15]$ for a detailed description of these models. The dynamic-slope models have been shown to offer a good description of sedimentation and erosion processes for large time scales (greater than $10^{4} \mathrm{y}$ ) and basin space scales (greater than $1 \mathrm{~km}$ ).

We consider here a dynamic-slope model simulating the evolution of a sedimentary basin in which sediments are modeled as a mixture of several lithologies $i=1, \ldots, L$ characterized by different grain size populations. The surficial transport process is a multi-lithology diffusive model introduced in [12], for which the fluxes are proportional to the slope of the topography and to a lithology fraction $c_{i}^{s}$ of the sediments at the surface of the basin (see also [5,9]). In the sequel, a simplified model is considered for which the diffusion coefficients are taken equal to one. It results that the sediment thickness $h$ is decoupled from the other unknowns of the system (i.e. for each lithology, the surface concentration $c_{i}^{s}$, and the concentration $c_{i}$ in lithology $i$ of the sediments in the basin), and satisfies a linear parabolic equation.

The remaining equations accounting for the mass conservation of the lithologies couple, for all $i=1, \ldots, L$, a first order linear equation for the surface concentration $c_{i}^{s}$ and a linear advection equation for the basin concentration $c_{i}$ for which $c_{i}^{s}$ appears as an input boundary condition at the top of the basin.

In [4], a weak formulation of (2.7) has been introduced (recalled in Def. 2.2) in order to cope with the difficulty to define the trace of the basin concentration $c_{i}$ at the top of the basin. In this previous article, the system has been discretized by an implicit integration in time and a cell centered finite volume scheme in space which has been shown to converge to a weak solution up to a subsequence.

The convergence of the numerical scheme proves the existence of a weak solution. The main objective of this article is to prove that this solution is unique, which will also yield the convergence of the full sequence of approximate solutions to the weak solution. This result is stated in Theorem 2.3 below.

The proof uses the linearity of the system (2.7) in the concentration unknowns $c_{i}$ and $c_{i}^{s}$, as well as the adjoint equations for which existence of a weak solution is obtained using the convergence of a numerical scheme. The core of the proof is derived in Section 4 and uses three lemmae which are proved in the subsequent sections. The numerical scheme for the adjoint equation and its convergence to a weak solution up to a subsequence is given in Section 5. The proof of this convergence is an adaptation of the one given in [4] for the direct problem, so only the main differences will be detailed. The main new difficulty to prove the uniqueness lies in two lemmae stating integration by part results for non smooth solutions of the adjoint and direct systems. The proof of these lemmae are detailed in Section 6 for the linear advection direct and adjoint equations and in Section 7 for the linear first order direct and adjoint equations.

The remaining of the paper outlines as follows. The mathematical model and its weak formulation are defined in Section 2, and the fully implicit finite volume discretization from [5] or [4] is recalled in Section 3.

\section{Mathematical MODEL AND WeAK FORMUlation}

A basin model specifies the geometry defined by the basin horizontal extension, the position of its base due to vertical tectonics displacements, and the sea level variations. It provides a description of the sediments considered as a mixture of different lithologies such as sand or shale. Finally it specifies the sediment transport laws and their coupling, as well as the sediment fluxes at the boundary of the basin (boundary conditions).

In this paper, the multi-lithology diffusion model described in $[5,9,12]$ is studied in a simplified case for which the diffusion coefficients of the lithologies are equal (to one to fix ideas). Also, for the sake of simplicity, the tectonics displacements, as well as the sea level variations, are not taken into account in the sequel.

The projection of the basin on a reference horizontal plane is considered as a fixed domain $\Omega \subset \mathbb{R}^{d}$, defining the horizontal extension of the basin, with $d=1$ for two dimensional basin models and $d=2$ for three 
dimensional models. Throughout this article, the symbols $\nabla$ and div denote respectively the gradient and the divergence operator in $\mathbb{R}^{d}$.

We denote by $h$ the sediment thickness unknown defined on the domain $\Omega \times \mathbb{R}_{+}^{*}$, and by $\mathcal{B}$ the domain

$$
\mathcal{B}=\left\{(x, z, t) \mid(x, t) \in \Omega \times \mathbb{R}_{+}^{*}, z<h(x, t)\right\}
$$

The sediments are modeled as a mixture of $L$ lithologies characterized by their grain size population. Each lithology, $i=1, \ldots, L$, is considered as an incompressible material of constant grain density and null porosity. On each point of the basin, the mixture is described by its composition given by the concentrations $c_{i}, i=1, \ldots, L$, defined on $\mathcal{B}$, and such that $c_{i} \geq 0$ for $i=1, \ldots, L$ and $\sum_{i=1}^{L} c_{i}=1$.

The model assumes that the sediment fluxes are nonzero only at the surface of the basin $(i . e$. for $z=h)$. The sediments transported by these surficial fluxes, i.e. which are deposited at the surface of the basin in case of sedimentation, or which pass through the surface in case of erosion, are characterized by their concentrations denoted by $c_{i}^{s}$, defined on $\Omega \times \mathbb{R}_{+}^{*}$, and such that $c_{i}^{s} \geq 0$ for $i=1, \ldots, L$ and $\sum_{i=1}^{L} c_{i}^{s}=1$.

Since the compaction is not considered, no change in time of the concentration $c_{i}$ can occur inside the basin. It results that $\partial_{t} c_{i}=0$ on $\mathcal{B}$. The evolution of $c_{i}$ is governed by the boundary condition at the top of the basin stating that $\left.c_{i}\right|_{z=h}=c_{i}^{s}$ in the case of sedimentation $\partial_{t} h>0$. Let $\mathcal{D}^{+}$denote the domain

$$
\mathcal{D}^{+}=\left\{(x, t) \in \Omega \times \mathbb{R}_{+}^{*} \mid \partial_{t} h(x, t)>0\right\},
$$

then, $c_{i}$ satisfies the conservation equation:

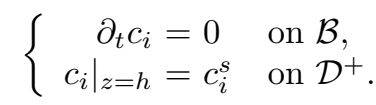

The conservation of the thickness fraction in lithology $i$

$$
\mathcal{M}_{i}(x, t)=\int_{0}^{h(x, t)} c_{i}(x, z, t) \mathrm{d} z,
$$

with $\sum_{i=1}^{L} \mathcal{M}_{i}=h$, states that for all $i=1, \ldots, L$

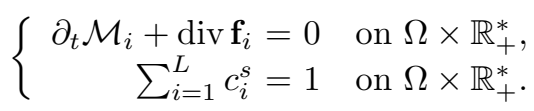

In the multi-lithology diffusive model described in [12], the flux $\mathbf{f}_{i}$ is proportional to the gradient of the topography $h$ and to the concentration $c_{i}^{s}$, with a diffusion coefficient $k_{i}$. In the sequel, we shall restrict ourselves to the simplified case $k_{i}=1$ for all $i=1, \ldots, L$, i.e. $\mathbf{f}_{i}:=-c_{i}^{s} \nabla h$, so that the sediment thickness $h$ decouples from the concentrations and satisfies a linear parabolic equation (see (2.6)). This assumption means physically that the lithologies are supposed to have the same transport properties. In such a case, the composition inside the basin is determined by the composition of the initial and input boundary sediments.

Neumann boundary conditions are imposed to $h$ on $\partial \Omega \times \mathbb{R}_{+}^{*}$,

$$
\nabla h \cdot \mathbf{n}=g \text { on } \partial \Omega \times \mathbb{R}_{+}^{*},
$$

with $\mathbf{n}$ the unit normal vector to $\partial \Omega$, outward to $\Omega$, and Dirichlet boundary conditions are prescribed to the surface concentrations

$$
c_{i}^{s}=\tilde{c}_{i} \text { on } \Sigma^{+}
$$

with

$$
\Sigma^{+}=\left\{(x, t) \in \partial \Omega \times \mathbb{R}_{+}^{*} \mid g(x, t)>0\right\},
$$

$\tilde{c}_{i} \geq 0$ for all $i=1, \ldots, L$ and $\sum_{i=1}^{L} \tilde{c}_{i}=1$. 
Initial conditions are prescribed to the sediment thickness stating that $\left.h\right|_{t=0}=h^{0}$ on $\Omega$, and to the basin concentrations stating that $\left.c_{i}\right|_{t=0}=c_{i}^{0}$ on the domain $\left\{(x, z) \mid x \in \Omega, z<h^{0}(x)\right\}$, with $c_{i}^{0} \geq 0$ for all $i=1, \ldots, L$ and $\sum_{i=1}^{L} c_{i}^{0}=1$.

In the following, we shall consider the new coordinate system for which the vertical position of a point in the basin is measured downward from the top of the basin, i.e. given by the change of variable $(x, \xi, t)=$ $\left(x^{\prime}, h\left(x^{\prime}, t^{\prime}\right)-z, t^{\prime}\right)$. In this coordinate system, let us consider the new unknown

$$
u_{i}(x, \xi, t)=c_{i}(x, h(x, t)-\xi, t) \text { defined for all }(x, \xi, t) \in \Omega \times \mathbb{R}_{+}^{*} \times \mathbb{R}_{+}^{*},
$$

and the initial condition

$$
u_{i}^{0}(x, \xi)=c_{i}^{0}\left(x, h^{0}(x)-\xi, t\right) \text { defined for all }(x, \xi) \in \Omega \times \mathbb{R}_{+}^{*} .
$$

Gathering all the equations, we obtain the following multi-lithology diffusive model:

$$
\begin{gathered}
\text { Surface conservations: }\left\{\begin{aligned}
\left.u_{i}\right|_{\xi=0} \partial_{t} h+\operatorname{div}\left(-c_{i}^{s} \nabla h\right)=0 & \text { on } \Omega \times \mathbb{R}_{+}^{*}, \\
\sum_{i=1}^{L} c_{i}^{s}=1 & \text { on } \Omega \times \mathbb{R}_{+}^{*}, \\
\left.\nabla h \cdot \mathbf{n}\right|_{\partial \Omega \times \mathbb{R}_{+}^{*}}=g & \text { on } \partial \Omega \times \mathbb{R}_{+}^{*}, \\
\left.c_{i}^{s}\right|_{\Sigma^{+}}=\tilde{c}_{i} & \text { on } \Sigma^{+}, \\
\left.h\right|_{t=0}=h^{0} & \text { on } \Omega,
\end{aligned}\right. \\
\text { Column conservations: }\left\{\begin{aligned}
\partial_{t} u_{i}+\partial_{t} h \partial_{\xi} u_{i}=0 & \text { on } \Omega \times \mathbb{R}_{+}^{*} \times \mathbb{R}_{+}^{*}, \\
\left.u_{i}\right|_{\xi=0}=c_{i}^{s} & \text { on } \mathcal{D}^{+}, \\
\left.u_{i}\right|_{t=0}=u_{i}^{0} & \text { on } \Omega \times \mathbb{R}_{+}^{*},
\end{aligned}\right.
\end{gathered}
$$

where we have taken into account the equality $\partial_{t} \mathcal{M}_{i}=\left.u_{i}\right|_{\xi=0} \partial_{t} h$ on $\Omega \times \mathbb{R}_{+}^{*}$ which derives formally from the definition (2.2) and the equation $\partial_{t} c_{i}=0$ on $\mathcal{B}$.

For this simplified model, summing equations $(2.4)$ over $i=1, \ldots, L$, it is clear that the sediment thickness $h$ satisfies the parabolic equation

$$
\left\{\begin{aligned}
\partial_{t} h-\Delta h & =0 & & \text { on } \Omega \times \mathbb{R}_{+}^{*}, \\
\left.\nabla h \cdot \mathbf{n}\right|_{\partial \Omega \times \mathbb{R}_{+}^{*}} & =g & & \text { on } \partial \Omega \times \mathbb{R}_{+}^{*}, \\
\left.h\right|_{t=0} & =h^{0} & & \text { on } \Omega,
\end{aligned}\right.
$$

while the concentrations $\left(c_{i}^{s}, u_{i}\right)$ verify, for each $i=1, \ldots, L$, the system of equations

$$
\left\{\begin{array}{rlrl}
\left.u_{i}\right|_{\xi=0} \partial_{t} h+\operatorname{div}\left(-c_{i}^{s} \nabla h\right)=0 & & \text { on } \Omega \times \mathbb{R}_{+}^{*} \\
\left.c_{i}^{s}\right|_{\Sigma^{+}} & =\tilde{c}_{i} & & \text { on } \Sigma^{+} \\
\partial_{t} u_{i}+\partial_{t} h \partial_{\xi} u_{i} & =0 & & \text { on } \Omega \times \mathbb{R}_{+}^{*} \times \mathbb{R}_{+}^{*} \\
\left.u_{i}\right|_{\xi=0} & =c_{i}^{s} & & \text { on } \mathcal{D}^{+} \\
\left.u_{i}\right|_{t=0} & =u_{i}^{0} & & \text { on } \Omega \times \mathbb{R}_{+}^{*} .
\end{array}\right.
$$

In the sequel, the following assumptions are made on the data.

\section{Hypothesis 2.1.}

(i) $\Omega$ is an open bounded subset of $\mathbb{R}^{d}$, of class $\mathrm{C}^{\infty}$;

(ii) $h^{0} \in \mathrm{C}^{2}(\bar{\Omega})$;

(iii) $g \in \mathrm{C}^{1}\left(\partial \Omega \times \mathbb{R}_{+}\right) \cap \mathrm{L}^{2}\left(\partial \Omega \times \mathbb{R}_{+}\right)$;

(iv) $g$ and $h^{0}$ are chosen according to the assumptions of Theorem 5.3 of [11] (p. 320) so that the unique solution $h$ of (2.6) is in $\mathrm{C}^{2}(\bar{\Omega} \times[0, T])$ for all $T>0$; 
(v) $\tilde{c}_{i} \in \mathrm{L}^{\infty}\left(\Sigma^{+}\right)$with $\tilde{c}_{i} \geq 0$ for $i=1, \ldots, L$ and $\sum_{i=1}^{L} \tilde{c}_{i}=1$;

(vi) $u_{i}^{0} \in \mathrm{L}^{\infty}\left(\Omega \times \mathbb{R}_{+}^{*}\right), u_{i}^{0} \geq 0$ for $i=1, \ldots, L$ and $\sum_{i=1}^{L} u_{i}^{0}=1$;

(vii) For all $T>0$, the boundaries $\partial \Sigma_{T}^{+}$and $\partial \Sigma_{T}^{-}$of the sets $\Sigma_{T}^{+}=\{(x, t) \in \partial \Omega \times(0, T) \mid g(x, t)>0\}$ and $\Sigma_{T}^{-}=\{(x, t) \in \partial \Omega \times(0, T) \mid g(x, t)<0\}$ are the union of a finite number of $\mathrm{C}^{1}$ manifolds of dimension at most $d-1$;

(viii) For all $T>0$, the boundaries $\partial \mathcal{D}_{T}^{+}$and $\partial \mathcal{D}_{T}^{-}$of the sets $\mathcal{D}_{T}^{+}=\left\{(x, t) \in \Omega \times(0, T) \mid \partial_{t} h(x, t)>0\right\}$, and $\mathcal{D}_{T}^{-}=\left\{(x, t) \in \Omega \times(0, T) \mid \partial_{t} h(x, t)<0\right\}$ are the union of a finite number of $\mathrm{C}^{1}$ manifolds of dimension at most d.

In the following, we shall denote by $\mathrm{C}_{c}^{\infty}\left(\mathbb{R}^{n}\right)$ the space of real valued functions

$$
\left\{\varphi \in \mathrm{C}^{\infty}\left(\mathbb{R}^{n}\right) \mid \operatorname{Supp}(\varphi) \text { bounded in } \mathbb{R}^{n}\right\}
$$

To obtain a rigorous mathematical formulation of (2.7), we are looking for weak solutions defined as follows for all $i=1, \ldots, L$.

Definition 2.2. Let us assume that Hypothesis 2.1 holds, and let $h$ denote the solution of problem (2.6). Then $\left(u_{i}, c_{i}^{s}\right) \in \mathrm{L}^{\infty}\left(\Omega \times \mathbb{R}_{+}^{*} \times \mathbb{R}_{+}^{*}\right) \times \mathrm{L}^{\infty}\left(\Omega \times \mathbb{R}_{+}^{*}\right)$ is said to be a weak solution of $(2.7)$ if it satisfies:

(i) for all $\varphi \in\left\{\phi \in \mathrm{C}_{c}^{\infty}\left(\mathbb{R}^{d+2}\right) \mid \phi(., 0,)=\right.$.0 on $\left.\Omega \times \mathbb{R}_{+}^{*} \backslash \mathcal{D}^{+}\right\}$

$$
\begin{gathered}
\int_{\Omega} \int_{\mathbb{R}_{+}} \int_{\mathbb{R}_{+}}\left[\partial_{t} \varphi(x, \xi, t)+\partial_{t} h(x, t) \partial_{\xi} \varphi(x, \xi, t)\right] u_{i}(x, \xi, t) \mathrm{d} t \mathrm{~d} \xi \mathrm{d} x \\
+\int_{\Omega} \int_{\mathbb{R}_{+}} u_{i}^{0}(x, \xi) \varphi(x, \xi, 0) \mathrm{d} \xi \mathrm{d} x+\int_{\Omega} \int_{\mathbb{R}_{+}} \partial_{t} h(x, t) c_{i}^{s}(x, t) \varphi(x, 0, t) \mathrm{d} t \mathrm{~d} x=0 ;
\end{gathered}
$$

(ii) for all $\psi \in\left\{\phi \in \mathrm{C}_{c}^{\infty}\left(\mathbb{R}^{d+2}\right) \mid \phi(., 0,)=\right.$.0 on $\left.\partial \Omega \times \mathbb{R}_{+}^{*} \backslash \Sigma^{+}\right\}$

$$
\begin{gathered}
-\int_{\Omega} \int_{\mathbb{R}_{+}} \int_{\mathbb{R}_{+}}\left[\partial_{t} \psi(x, \xi, t)+\partial_{t} h(x, t) \partial_{\xi} \psi(x, \xi, t)\right] u_{i}(x, \xi, t) \mathrm{d} t \mathrm{~d} \xi \mathrm{d} x-\int_{\Omega} \int_{\mathbb{R}_{+}} u_{i}^{0}(x, \xi) \psi(x, \xi, 0) \mathrm{d} \xi \mathrm{d} x \\
\quad+\int_{\mathbb{R}_{+}}\left(\int_{\Omega} c_{i}^{s}(x, t) \nabla h(x, t) \cdot \nabla \psi(x, 0, t) \mathrm{d} x-\int_{\partial \Omega} \tilde{c}_{i}(x, t) g(x, t) \psi(x, 0, t) \mathrm{d} \gamma(x)\right) \mathrm{d} t=0
\end{gathered}
$$

The main objective of this article is to prove the following theorem.

Theorem 2.3. Assuming that Hypothesis 2.1 holds, for all $i=1, \ldots, L$ there exists a weak solution $\left(u_{i}, c_{i}^{s}\right)$ to problem (2.7) in the sense of Definition 2.2 satisfying $\sum_{i=1}^{L} u_{i}=1, u_{i} \geq 0$, and $\sum_{i=1}^{L} c_{i}^{s}=1, c_{i}^{s} \geq 0$. Moreover, for all $i=1, \ldots, L$, the weak solution $u_{i}$ in the sense of Definition 2.2 is unique.

Existence of a weak solution $\left(u_{i}, c_{i}^{s}\right)$ has already been proved in [4] using the convergence of the numerical scheme recalled in the next section. The proof of uniqueness will be obtained using the existence of a weak solution to the adjoint system described in Section 4 and two integration by part technical lemmae the proof of which is detailed in Sections 6 and 7. The existence of the adjoint weak solution is proved in Section 5 using the convergence of a numerical scheme in a very similar way as in [4].

Remark 2.4. Existence and uniqueness still hold when considering a compaction model given by a depth porosity relation $\Phi(h-z)$ or/and when considering a non linear diffusion coefficient $k_{i}=k(h)$ for all $i=1, \ldots, L$. The main difference is that $h$ denotes the solution of a non linear parabolic equation of the form

$$
\partial_{t} h-\Delta \Psi(h)=0,
$$

with $\Psi$ a strictly increasing smooth function and $\Psi^{\prime}$ is bounded from below by a strictly positive constant and bounded from above. 


\section{Finite volume scheme}

In this section the numerical scheme described in [5] and [4] is recalled.

The system (2.4)-(2.5) is discretized by a fully implicit time integration and a finite volume method with cell centered variables. We shall consider in the sequel admissible meshes according to the following definition.

Definition 3.1 (admissible meshes). Let $\Omega$ be a bounded domain of $\mathbb{R}^{d}, d=1$ or 2 . An admissible finite volume mesh of $\Omega$ for the discretization of problem (2.4)-(2.5) is given by a family of "control volumes", denoted by $\mathcal{K}$, which are open disjoint subsets of $\Omega$, and a family of points of $\Omega$, denoted by $\mathcal{P}$, satisfying the following properties:

(i) The closure of the union of all the control volumes of $\mathcal{K}$ is $\bar{\Omega}$.

(ii) For any $\kappa, \kappa^{\prime} \in \mathcal{K}$ with $\kappa \neq \kappa^{\prime}$, either the $(d-1)$-dimensional measure of $\bar{\kappa} \cap \bar{\kappa}^{\prime}$, denoted by $m\left(\bar{\kappa} \cap \bar{\kappa}^{\prime}\right)$, is null, or it is strictly positive and $\bar{\kappa} \cap \bar{\kappa}^{\prime}$ is included in an hyperplane of $\mathbb{R}^{d}$. In the following, we will denote by $\Sigma_{i n t}$ the family of subsets $\sigma$ of $\Omega$ contained in hyperplanes of $\mathbb{R}^{d}$ with strictly positive measures, and such that there exist $\kappa, \kappa^{\prime} \in \mathcal{K}$ with $m\left(\bar{\kappa} \cap \bar{\kappa}^{\prime}\right)>0$ and $\bar{\sigma}=\bar{\kappa} \cap \bar{\kappa}^{\prime}$. We shall also denote by $\kappa \mid \kappa^{\prime} \in \Sigma_{\text {int }}$ the edge between the cells $\kappa$ and $\kappa^{\prime}$.

(iii) The family $\mathcal{P}=\left(x_{\kappa}\right)_{\kappa \in \mathcal{K}}$ is such that $x_{\kappa} \in \bar{\kappa}$ (for any $\kappa \in \mathcal{K}$ ) and, if $\sigma=\kappa \mid \kappa^{\prime} \in \Sigma_{\text {int }}$, it is assumed that $x_{\kappa} \neq x_{\kappa^{\prime}}$ and that the straight line going through $x_{\kappa}$ and $x_{\kappa^{\prime}}$ is orthogonal to the edge $\sigma$.

(iv) For any $\kappa \in \mathcal{K}$, there exists a subset $\Sigma_{\kappa}$ of $\Sigma_{\text {int }}$ such that $\partial \kappa \backslash \partial \Omega=\bar{\kappa} \backslash(\kappa \cup \partial \Omega)=\cup_{\sigma \in \Sigma_{\kappa}} \bar{\sigma}$.

We shall denote by $\left(\mathcal{K}, \Sigma_{\text {int }}, \mathcal{P}\right)$ this admissible mesh.

Note that, in this definition, no assumption is made on the boundary edges of the mesh.

Let $\left(\mathcal{K}, \Sigma_{\text {int }}, \mathcal{P}\right)$ be an admissible mesh of $\Omega$ in the sense of Definition 3.1. In the sequel, $\delta \mathcal{K}=\sup \{\operatorname{diam}(\kappa), \kappa \in$ $\mathcal{K}\}$ will denote the mesh size of $\left(\mathcal{K}, \Sigma_{i n t}, \mathcal{P}\right),|\kappa|$ is the $d$-dimensional Lebesgue measure of the cell $\kappa, \mathcal{K}_{\kappa}$ the set of neighboring cells of $\kappa$ (excluding $\kappa),|\sigma|$ (resp. $|\partial \kappa \cap \partial \Omega|$ ) the $(d-1)$-dimensional Lebesgue measure of the edge $\sigma$ (resp. of $\partial \kappa \cap \partial \Omega), T_{\kappa \kappa^{\prime}}=T_{\sigma}$ the transmissibility of the edge $\sigma=\kappa \mid \kappa^{\prime}$, defined by $T_{\kappa \kappa^{\prime}}:=\frac{|\sigma|}{d\left(\kappa, \kappa^{\prime}\right)}$ with $d\left(\kappa, \kappa^{\prime}\right)$ the distance between the points $x_{\kappa}$ and $x_{\kappa^{\prime}}, \operatorname{reg}(\mathcal{K})$ the geometrical factor defined by $\operatorname{reg}(\mathcal{K})=\max _{\substack{\sigma \in \sum_{i n t} \\ \sigma=\kappa \mid \kappa^{\prime}}} \frac{\delta \mathcal{K}}{d\left(\kappa, \kappa^{\prime}\right)}$, and $\mathbf{n}_{\kappa \kappa^{\prime}}$ the unit normal vector to $\sigma=\kappa \mid \kappa^{\prime}$ outward to $\kappa$.

For a given set $\mathcal{P}$ of disjoint points of $\Omega$, an example of such an admissible mesh is the Voronoï mesh defined by

$$
\kappa=\left\{x \in \Omega, d\left(x, x_{\kappa}\right)<d\left(x, x_{\kappa^{\prime}}\right) \text { for all } x_{\kappa^{\prime}} \in \mathcal{P}, x_{\kappa^{\prime}} \neq x_{\kappa}\right\} .
$$

For any set $A$, we shall also denote by $\chi_{\mathrm{A}}$ the function such that $\chi_{\mathrm{A}}(y)=1$ if $y \in A$ and $\chi_{\mathrm{A}}(y)=0$ otherwise. Finally, for any function $f$, let us define $f^{+}=\max (f, 0) \geq 0, f^{-}=-\min (f, 0) \geq 0$, such that $f=f^{+}-f^{-}$, and $|f|=f^{+}+f^{-}$.

The time discretization is denoted by $t^{n}, n \in \mathbb{N}$, such that $t^{0}=0$ and $\Delta t^{n+1}=t^{n+1}-t^{n}>0$. In the following, the superscript $n, n \in \mathbb{N}$, will be used to denote that the unknowns are considered at time $t^{n}$. Assuming that the set $\left\{\Delta t^{n} \mid n \in \mathbb{N}\right\}$ is bounded, let $\Delta t$ denote $\sup \left\{\Delta t^{n} \mid n \in \mathbb{N}\right\}$ and, for a given $T>0$, let $N_{\Delta t}$ be the integer such that $t^{N_{\Delta t}}<T \leq t^{N_{\Delta t}+1}$.

Let us now recall the discretization of (2.4)-(2.5) already introduced in [5]. For all control volumes $\kappa \in \mathcal{K}$, the following initial values are defined:

(1) $h_{\kappa}^{0}$ is the initial approximation of $h$ in $\kappa$ defined by $h_{\kappa}^{0}=h^{0}\left(x_{\kappa}\right)$; 
(2) $u_{i, \kappa}^{0}$, for all species $i$, is the approximation of $u_{i}^{0}$ on the cell $\kappa$, defined by $u_{i, \kappa}^{0}(\xi)=\frac{1}{|\kappa|} \int_{\kappa} u_{i}^{0}(x, \xi) \mathrm{d} x$ for $\xi \in \mathbb{R}_{+}^{*}$, and let $c_{i, \kappa}^{0}$ be defined on $\left(-\infty, h_{\kappa}^{0}\right)$ by $c_{i, \kappa}^{0}(z)=u_{i, \kappa}^{0}\left(h_{\kappa}^{0}-z\right)$.

We now give a discretization of equations (2.4)-(2.5) within a given control volume $\kappa \in \mathcal{K}$ between times $t^{n}$ and $t^{n+1}$ :

Conservation of surface sediments:

$$
\begin{gathered}
\frac{\Delta \mathcal{M}_{i, \kappa}^{n+1}}{\Delta t^{n+1}}|\kappa|+\sum_{\kappa^{\prime} \in \mathcal{K}_{\kappa}} c_{i, \kappa \kappa^{\prime}}^{s, n+1} T_{\kappa \kappa^{\prime}}\left(h_{\kappa}^{n+1}-h_{\kappa^{\prime}}^{n+1}\right)-|\partial \kappa \cap \partial \Omega| \tilde{c}_{i, \kappa}^{n+1} g_{\kappa}^{(+), n+1}+|\partial \kappa \cap \partial \Omega| c_{i, \kappa}^{s, n+1} g_{\kappa}^{(-), n+1}=0, \\
\sum_{i=1}^{L} c_{i, \kappa}^{s, n+1}=1 .
\end{gathered}
$$

Conservation of column sediments:

$$
\begin{aligned}
\text { if } h_{\kappa}^{n+1} \geq h_{\kappa}^{n}, \quad\left\{\begin{array}{l}
\Delta \mathcal{M}_{i, \kappa}^{n+1}=c_{i, \kappa}^{s, n+1}\left(h_{\kappa}^{n+1}-h_{\kappa}^{n}\right) \\
c_{i, \kappa}^{n+1}(z)=c_{i, \kappa}^{n}(z), z<h_{\kappa}^{n} \\
c_{i, \kappa}^{n+1}(z)=c_{i, \kappa}^{s, n+1}, z \in\left(h_{\kappa}^{n}, h_{\kappa}^{n+1}\right)
\end{array}\right. \\
\text { else }\left\{\begin{array}{c}
\Delta \mathcal{M}_{i, \kappa}^{n+1}=\int_{h_{\kappa}^{n}}^{h_{n}^{n+1}} c_{i, \kappa}^{n}(z) \mathrm{d} z \\
c_{i, \kappa}^{n+1}(z)=c_{i, \kappa}^{n}(z), z<h_{\kappa}^{n+1}
\end{array}\right.
\end{aligned}
$$

In (3.2)-(3.5), the following notation is used.

1. $h_{\kappa}^{n}$ is the approximation of the sediment thickness $h$ at time $t^{n}$ in $\kappa$;

2. $c_{i, \kappa}^{s, n+1}$ is the approximation of the surface sediment concentration $c_{i}^{s}$ at time $t^{n+1}$ in $\kappa$;

3. the function $c_{i, \kappa}^{n}$, defined on the column $\left(-\infty, h_{\kappa}^{n}\right)$, is the approximation of the sediment concentration in lithology $i$ in the column $\left\{(x, z) \mid x \in \kappa, z<h\left(x, t^{n}\right)\right\}$ at time $t^{n}$;

4. $c_{i, \kappa \kappa^{\prime}}^{s, n+1}$ is the upstream weighted evaluation of the surface sediment concentration in lithology $i$ at the edge $\sigma$ between the cells $\kappa$ and $\kappa^{\prime}$ with respect to the sign of $h_{\kappa}^{n+1}-h_{\kappa^{\prime}}^{n+1}$ :

$$
c_{i, \kappa \kappa^{\prime}}^{s, n+1}=\left\{\begin{array}{l}
c_{i, \kappa}^{s, n+1} \text { if } h_{\kappa}^{n+1}>h_{\kappa^{\prime}}^{n+1} \\
c_{i, \kappa^{\prime}}^{s, n+1} \text { otherwise }
\end{array}\right.
$$

5. $g_{\kappa}^{(+), n+1}$ and $g_{\kappa}^{(-), n+1}$ are the following approximations of the boundary fluxes $g^{+}$and $g^{-}$:

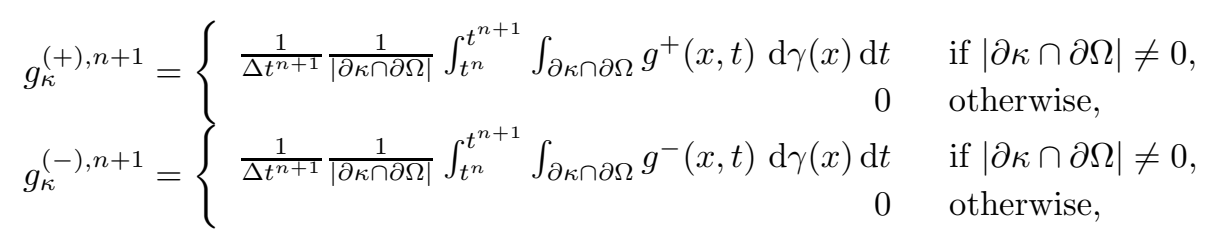

and consequently for all $\kappa \in \mathcal{K}$,

$$
g_{\kappa}^{n+1}=\frac{1}{\Delta t^{n+1}} \frac{1}{|\partial \kappa \cap \partial \Omega|} \int_{t^{n}}^{t^{n+1}} \int_{\partial \kappa \cap \partial \Omega} g(x, t) \mathrm{d} \gamma(x) \mathrm{d} t=g_{\kappa}^{(+), n+1}-g_{\kappa}^{(-), n+1}
$$


6. $\tilde{c}_{i, \kappa}^{n+1}$ is the approximation of $\tilde{c}_{i}$ extended by 0 on $\left(\partial \Omega \times \mathbb{R}_{+}^{*}\right) \backslash \Sigma^{+}$:

$$
\tilde{c}_{i, \kappa}^{n+1}=\left\{\begin{aligned}
\frac{1}{\Delta t^{n+1}} \frac{1}{|\partial \kappa \cap \partial \Omega|} \int_{t^{n}}^{t^{n+1}} \int_{\partial \kappa \cap \partial \Omega} \tilde{c}_{i}(x, t) \mathrm{d} \gamma(x) \mathrm{d} t & \text { if }|\partial \kappa \cap \partial \Omega| \neq 0 \\
0 & \text { otherwise }
\end{aligned}\right.
$$

and it results that $\tilde{c}_{i, \kappa}^{n+1} \in[0,1]$.

Considering the coordinate system $\xi=h_{\kappa}^{n}-z$, the function $u_{i, \kappa}^{n}$ is defined for all $\kappa \in \mathcal{K}, n \geq 0$ and $i=1, \ldots, L$, by

$$
u_{i, \kappa}^{n}(\xi)=c_{i, \kappa}^{n}\left(h_{\kappa}^{n}-\xi\right) \text { for all } \xi \in \mathbb{R}_{+}^{*} .
$$

For the sake of simplicity, it is assumed in the remaining of this article that $\Delta t=\Delta t^{n}$ for all $n \geq 1$, although all the results presented in the sequel readily extend to variable time steps.

In [4], we have proved, for all $n \geq 0$, the existence of solutions $\left(h_{\kappa}^{n}\right)_{\kappa \in \mathcal{K}},\left(c_{i, \kappa}^{s, n+1}\right)_{\kappa \in \mathcal{K}},\left(c_{i, \kappa}^{n}\right)_{\kappa \in \mathcal{K}}$ and $\left(u_{i, \kappa}^{n}\right)_{\kappa \in \mathcal{K}}$, $i=1, \ldots, L$, to problem $(3.2)-(3.6)$. These solutions are unique except for the surface concentrations $c_{i, \kappa}^{s, n+1}$ which are arbitrary ( $\operatorname{such}$ that $\left.\sum_{j=1}^{L} c_{j, \kappa}^{s, n+1}=1\right)$ at some degenerate points $(\kappa, n+1)$.

For any admissible mesh $\left(\mathcal{K}, \Sigma_{i n t}, \mathcal{P}\right)$ of $\Omega$ in the sense of Definition 3.1, any time step $\Delta t>0$, and $i=1, \ldots, L$, let $h_{\mathcal{K}, \Delta t}$ and $u_{i, \mathcal{K}, \Delta t}$, defined on $\Omega \times \mathbb{R}_{+}^{*}$ and $\Omega \times \mathbb{R}_{+}^{*} \times \mathbb{R}_{+}^{*}$, denote the functions such that

$$
\left\{\begin{aligned}
h_{\mathcal{K}, \Delta t}(x, t) & =h_{\kappa}^{n+1} \\
u_{i, \mathcal{K}, \Delta t}(x, \xi, t) & =u_{i, \kappa}^{n+1}(\xi)
\end{aligned}\right.
$$

for all $x \in \kappa, \kappa \in \mathcal{K}, t \in\left(t^{n}, t^{n+1}\right], n \geq 0, \xi \in \mathbb{R}_{+}^{*}$, where $h_{\kappa}^{n}, u_{i, \kappa}^{n}$ are the solutions of (3.2)-(3.6).

The following theorem is a straightforward corollary of both Theorem 2.3 and the theorem proved in [4] stating the convergence up to a subsequence of the approximate solutions to a weak solution in the sense of Definition 2.2.

Theorem 3.2. Hypothesis 2.1 is assumed to hold. For all $m \in \mathbb{N}$, let $\left(\mathcal{K}_{m}, \Sigma_{\text {int }}^{m}, \mathcal{P}_{m}\right)$ be an admissible mesh of $\Omega$ in the sense of Definition 3.1 and $\Delta t_{m}>0$. Let us assume that there exists $\alpha>0$ such that reg $\left(\mathcal{K}_{m}\right) \leq \alpha$ for all $m \in \mathbb{N}$, and that $\Delta t_{m} \rightarrow 0, \frac{\delta \mathcal{K}_{m}}{\sqrt{\Delta t_{m}}} \rightarrow 0$ as $m \rightarrow \infty$.

For all $m \in \mathbb{N}$ and $i=1, \ldots, L$, let $h_{\mathcal{K}_{m}, \Delta t_{m}}$ and $u_{i, \mathcal{K}_{m}, \Delta t_{m}}$ denote the unique solutions of (3.2)-(3.6) defined by (3.7) with $\mathcal{K}=\mathcal{K}_{m}, \Delta t=\Delta t_{m}$.

Then, the sequence $\left(h_{\mathcal{K}_{m}, \Delta t_{m}}\right)_{m \in \mathbb{N}}$ converges to the solution $h$ of problem $(2.6)$ in $\mathrm{L}^{\infty}\left(0, T ; \mathrm{L}^{2}(\Omega)\right)$ for all $T>0$, and the sequence $\left(u_{i, \mathcal{K}_{m}, \Delta t_{m}}\right)_{m \in \mathbb{N}}$ converges to the weak solution $u_{i}$ of $(2.7)$ in the sense of Definition 2.2

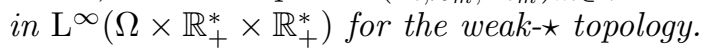

\section{Proof of Theorem 2.3}

To show the existence of a weak solution $\left(u_{i}, c_{i}^{s}\right), i=1, \ldots, L$, we apply the convergence of the numerical scheme (3.2)-(3.5) proved in [4] for a family of admissible meshes $\left(\mathcal{K}_{m}, \Sigma_{i n t}^{m}, \mathcal{P}_{m}\right)_{m \in \mathbb{N}}$ satisfying the assumptions of Theorem 3.2. To build such a family, let us consider for all $m \in \mathbb{N}$, the time step $\Delta t_{m}=\frac{1}{m+1}$, the set of points $\mathcal{P}_{m}=\left\{\frac{\mathbf{k}}{m+1}, \mathbf{k} \in \mathbb{Z}^{d}\right\} \cap \Omega$, and let $\mathcal{K}_{m}$ be the Voronoï mesh (3.1) obtained from the set $\mathcal{P}_{m}$. Using the smoothness of the bounded domain $\Omega$, one can check that this family of meshes satisfies all the assumptions of Theorem 3.2.

The main objective of this article is now to prove the uniqueness of the weak solution $u_{i}, i=1, \ldots, L$. For any given surface concentration $c_{i}^{s} \in \mathrm{L}^{\infty}\left(\Omega \times \mathbb{R}_{+}^{*}\right)$, we need to study the weak formulation (2.8) of the linear advection equation $\partial_{t} u_{i}+\partial_{t} h \partial_{\xi} u_{i}=0$ with the input boundary condition $c_{i}^{s}$ on $\mathcal{D}^{+}$and the initial condition $u_{i}^{0}$. 
We also need to prove an integration by part formula for the solutions of this equation and its adjoint equation. These results are the purpose of the following lemma, the proof of which is postponed to section 6 .

In the sequel, $\mathcal{L}$ will denote the operator $\mathcal{L}=\partial_{t}+\partial_{t} h \partial_{\xi}$.

Lemma 4.1. Hypothesis 2.1 is assumed to hold. Then, for any time $T>0$, any functions $f \in \mathrm{L}^{\infty}\left(\Omega \times \mathbb{R}_{+}^{*} \times\right.$ $(0, T)), l^{s} \in \mathrm{L}^{\infty}\left(\mathcal{D}_{T}^{+}\right)$, and $v^{0} \in \mathrm{L}^{\infty}\left(\Omega \times \mathbb{R}_{+}^{*}\right)$, the equation

$$
\left\{\begin{aligned}
\mathcal{L} v=f & & \text { on } \Omega \times \mathbb{R}_{+}^{*} \times(0, T), \\
\left.v\right|_{\xi=0}=l^{s} & & \text { on } \mathcal{D}_{T}^{+}, \\
\left.v\right|_{t=0}=v^{0} & & \text { on } \Omega \times \mathbb{R}_{+}^{*},
\end{aligned}\right.
$$

has a unique weak solution in $\mathrm{L}^{\infty}\left(\Omega \times \mathbb{R}_{+}^{*} \times(0, T)\right)$ in the sense that for all $\varphi \in\left\{\phi \in \mathrm{C}_{c}^{\infty}\left(\mathbb{R}^{d+2}\right) \mid \phi(., 0,)=\right.$. 0 on $\Omega \times(0, T) \backslash \mathcal{D}_{T}^{+}$and $\phi(., ., T)=0$ on $\left.\Omega \times \mathbb{R}_{+}^{*}\right\}$, one has

$$
\begin{gathered}
\int_{\Omega} \int_{\mathbb{R}_{+}} \int_{0}^{T}((\mathcal{L} \varphi)(x, \xi, t) v(x, \xi, t)+f(x, \xi, t) \varphi(x, \xi, t)) \mathrm{d} t \mathrm{~d} \xi \mathrm{d} x \\
+\int_{\Omega} \int_{\mathbb{R}_{+}} v^{0}(x, \xi) \varphi(x, \xi, 0) \mathrm{d} \xi \mathrm{d} x+\int_{\Omega} \int_{0}^{T} \partial_{t} h(x, t) l^{s}(x, t) \varphi(x, 0, t) \mathrm{d} t \mathrm{~d} x=0 .
\end{gathered}
$$

The weak solution $v$ of (4.1) has a trace on $t=T$ in $\mathrm{L}^{\infty}\left(\Omega \times \mathbb{R}_{+}^{*}\right)$, and the function $v \partial_{t} h$ has a trace on $\xi=0$ in $\mathrm{L}^{\infty}(\Omega \times(0, T))$, such that for any $\varphi \in \mathrm{C}_{c}^{\infty}\left(\mathbb{R}^{d+2}\right)$ one has

$$
\begin{gathered}
\int_{\Omega} \int_{\mathbb{R}_{+}} \int_{0}^{T}((\mathcal{L} \varphi)(x, \xi, t) v(x, \xi, t)+f(x, \xi, t) \varphi(x, \xi, t)) \mathrm{d} t \mathrm{~d} \xi \mathrm{d} x \\
+\int_{\Omega} \int_{\mathbb{R}_{+}}(v(x, \xi, 0) \varphi(x, \xi, 0)-v(x, \xi, T) \varphi(x, \xi, T)) \mathrm{d} \xi \mathrm{d} x \\
\quad+\int_{\Omega} \int_{0}^{T} \partial_{t} h(x, t) v(x, 0, t) \varphi(x, 0, t) \mathrm{d} t \mathrm{~d} x=0 .
\end{gathered}
$$

Let $T>0$, and $w$ be the weak solution in $\mathrm{L}^{\infty}\left(\Omega \times \mathbb{R}_{+}^{*} \times(0, T)\right)$ of the adjoint equation

$$
\left\{\begin{array}{rlrl}
-\mathcal{L} w & =r & & \text { on } \Omega \times \mathbb{R}_{+}^{*} \times(0, T), \\
\left.w\right|_{\xi=0}=q^{s} & & \text { on } \mathcal{D}_{T}^{-} \\
\left.w\right|_{t=T} & =w^{T} & & \text { on } \Omega \times \mathbb{R}_{+}^{*},
\end{array}\right.
$$

defined in a similar way as above with $r \in \mathrm{L}^{\infty}\left(\Omega \times \mathbb{R}_{+}^{*} \times(0, T)\right)$ a compactly supported function on $\bar{\Omega} \times \mathbb{R}_{+} \times[0, T]$, $w^{T} \in \mathrm{L}^{\infty}\left(\Omega \times \mathbb{R}_{+}^{*}\right)$ a compactly supported function on $\bar{\Omega} \times \mathbb{R}_{+}$, and $q^{s} \in \mathrm{L}^{\infty}(\Omega \times(0, T))$. Then, one has

$$
\begin{gathered}
\int_{\Omega} \int_{\mathbb{R}_{+}} \int_{0}^{T}(v(x, \xi, t)(\mathcal{L} w)(x, \xi, t)+(\mathcal{L} v)(x, \xi, t) w(x, \xi, t)) \mathrm{d} t \mathrm{~d} \xi \mathrm{d} x \\
-\int_{\Omega} \int_{\mathbb{R}_{+}}(v(x, \xi, T) w(x, \xi, T)-v(x, \xi, 0) w(x, \xi, 0)) \mathrm{d} \xi \mathrm{d} x \\
\quad+\int_{\Omega} \int_{0}^{T} \partial_{t} h(x, t) v(x, 0, t) w(x, 0, t) \mathrm{d} t \mathrm{~d} x=0
\end{gathered}
$$

Let us denote by $\left(v_{i}, d_{i}^{s}\right)$ the difference between any two weak solutions of (2.7). From the linearity of the set of equations $(2.7)$, the functions $\left(v_{i}, d_{i}^{s}\right)$ satisfy the weak formulation (2.8)-(2.9) with homogeneous boundary and initial conditions. 
Let $T>0$, from Lemma 4.1 , the function $v_{i} \partial_{t} h$ has a trace at $\xi=0$ in $\mathrm{L}^{\infty}(\Omega \times(0, T))$ denoted by $\left.v_{i}\right|_{\xi=0} \partial_{t} h$. Then, from the integration by part formula (4.3) of Lemma 4.1 and the weak formulation (2.9), it results that for all $\varphi \in\left\{\phi \in \mathrm{C}_{c}^{\infty}\left(\mathbb{R}^{d+1}\right) \mid \phi(x, t)=0\right.$ on $\partial \Omega \times(0, T) \backslash \Sigma_{T}^{+}$, and $\phi(x, T)=0$ on $\left.\Omega\right\}$, one has

$$
\int_{\Omega} \int_{0}^{T} v_{i}(x, 0, t) \partial_{t} h(x, t) \varphi(x, t) \mathrm{d} t \mathrm{~d} x+\int_{\Omega} \int_{0}^{T} d_{i}^{s}(x, t) \nabla h(x, t) \cdot \nabla \varphi(x, t) \mathrm{d} t \mathrm{~d} x=0 .
$$

We deduce that

$$
\operatorname{div}\left(-d_{i}^{s} \nabla h\right)=-\left.v_{i}\right|_{\xi=0} \partial_{t} h \in \mathrm{L}^{\infty}(\Omega \times(0, T)) .
$$

Since $\partial_{t} h-\Delta h=0$ one has also

$$
\nabla h \cdot \nabla d_{i}^{s}=\left(\left.v_{i}\right|_{\xi=0}-d_{i}^{s}\right) \partial_{t} h \in \mathrm{L}^{\infty}(\Omega \times(0, T)) .
$$

Let us consider the adjoint system

$$
\left\{\begin{aligned}
-\left.w_{i}\right|_{\xi=0} \partial_{t} h+\operatorname{div}\left(q_{i}^{s} \nabla h\right) & =0 & & \text { on } \Omega \times(0, T), \\
\left.q_{i}^{s}\right|_{\Sigma_{T}^{-}} & =0 & & \text { on } \Sigma_{T}^{-}, \\
-\mathcal{L} w_{i} & =v_{i} & & \text { on } \Omega \times \mathbb{R}_{+}^{*} \times(0, T), \\
\left.w_{i}\right|_{\xi=0} & =q_{i}^{s} & & \text { on } \mathcal{D}_{T}^{-}, \\
\left.w_{i}\right|_{t=T} & =\left.v_{i}\right|_{t=T} & & \text { on } \Omega \times \mathbb{R}_{+}^{*} .
\end{aligned}\right.
$$

The following lemma states that there exists at least one weak solution $\left(w_{i}, q_{i}^{s}\right)$ in $\mathrm{L}^{\infty}\left(\Omega \times \mathbb{R}_{+}^{*} \times(0, T)\right) \times$ $\mathrm{L}^{\infty}(\Omega \times(0, T))$ to these adjoint equations defined similarly as in Definition 2.2 (see also Def. 5.2). The proof of this lemma uses the convergence of the numerical scheme described in Section 3 adapted to the case of a non vanishing right hand side $v_{i}$ in $\mathrm{L}^{\infty}\left(\Omega \times \mathbb{R}_{+}^{*} \times(0, T)\right)$. It is postponed to Section 5 .

Lemma 4.2. Hypothesis 2.1 is assumed to hold. Then, there exists at least one weak solution $\left(w_{i}, q_{i}^{s}\right)$ of $(4.9)$ defined in a similar way as in Definition 2.2 .

Considering such a weak solution, the following equation is derived as above

$$
\operatorname{div}\left(q_{i}^{s} \nabla h\right)=\left.w_{i}\right|_{\xi=0} \partial_{t} h \in \mathrm{L}^{\infty}(\Omega \times(0, T)) .
$$

From equations (4.8) and (4.10), the function $\operatorname{div}\left(q_{i}^{s} d_{i}^{s} \nabla h\right)$ is in $\mathrm{L}^{\infty}(\Omega \times(0, T))$. It results from Lemma 7.2 proved in Section 7 that the vector field $q_{i}^{s} d_{i}^{s} \nabla h$ has a normal trace in $\mathrm{L}^{\infty}(\partial \Omega \times(0, T))$. As formally $d_{i}^{s}$ vanishes on $\Sigma_{T}^{+}, q_{i}^{s}$ vanishes on $\Sigma_{T}^{-}$, and the normal trace $g$ of $\nabla h$ vanishes on $\partial \Omega \times(0, T) \backslash\left(\Sigma_{T}^{+} \cup \Sigma_{T}^{-}\right)$, the normal trace of $q_{i}^{s} d_{i}^{s} \nabla h$ vanishes on the boundary $\partial \Omega \times(0, T)$. This result is stated by the following lemma for which a rigorous proof will be given in section 7 .

Lemma 4.3. Hypothesis 2.1 is assumed to hold. Then, for any $T>0$, any weak solutions $\left(w_{i}, q_{i}^{s}\right)$ of the adjoint problem (4.9) and $\left(v_{i}, d_{i}^{s}\right)$ of problem (2.7) with homogeneous boundary and initial conditions, one has

$$
\int_{\Omega} \int_{0}^{T} \operatorname{div}\left(q_{i}^{s} d_{i}^{s} \nabla h\right) \mathrm{d} t \mathrm{~d} x=0
$$

Since the velocity $\partial_{t} h$ is uniformly bounded on $\bar{\Omega} \times[0, T]$ for any time $T>0$, the function $v_{i}$ (resp. its trace $\left.v_{i}\right|_{t=T}$ ) is compactly supported in $\bar{\Omega} \times \mathbb{R}_{+} \times[0, T]$ (resp. in $\bar{\Omega} \times \mathbb{R}_{+}$) (see also the definition of the characteristic solution of (2.7) in section 6). Applying the integration by part formula (4.5) of Lemma 4.1 to $v=v_{i}$ and $w=w_{i}$, we obtain that for any time $T>0$

$$
\int_{\Omega} \int_{\mathbb{R}_{+}} \int_{0}^{T}\left|v_{i}\right|^{2}(x, \xi, t) \mathrm{d} t \mathrm{~d} \xi \mathrm{d} x+\int_{\Omega} \int_{\mathbb{R}_{+}}\left|v_{i}\right|^{2}(x, \xi, T) \mathrm{d} \xi \mathrm{d} x=\int_{\Omega} \int_{0}^{T} \partial_{t} h(x, t) v_{i}(x, 0, t) w_{i}(x, 0, t) \mathrm{d} t \mathrm{~d} x .
$$


From Lemma 4.3 and the integration over $\Omega \times(0, T)$ of equation $(4.10)$ multiplied by $d_{i}^{s}$, it results that

$$
\int_{\Omega} \int_{0}^{T} d_{i}^{s}(x, t) w_{i}(x, 0, t) \partial_{t} h(x, t) \mathrm{d} t \mathrm{~d} x+\int_{\Omega} \int_{0}^{T} q_{i}^{s}(x, t) \nabla d_{i}^{s}(x, t) \cdot \nabla h(x, t) \mathrm{d} t \mathrm{~d} x=0 .
$$

Also, multiplying equation (4.8) by $q_{i}^{s}$ and integrating over $\Omega \times(0, T)$, we obtain that

$$
\int_{\Omega} \int_{0}^{T}\left(v_{i}(x, 0, t)-d_{i}^{s}(x, t)\right) q_{i}^{s}(x, t) \partial_{t} h(x, t) \mathrm{d} t \mathrm{~d} x-\int_{\Omega} \int_{0}^{T} q_{i}^{s}(x, t) \nabla d_{i}^{s}(x, t) \cdot \nabla h(x, t) \mathrm{d} t \mathrm{~d} x=0
$$

Summing equations (4.13) and (4.14) and taking into account the boundary conditions $\left.w_{i}\right|_{\xi=0}=q_{i}^{s}$ on $\mathcal{D}_{T}^{-}$, $\left.v_{i}\right|_{\xi=0}=d_{i}^{s}$ on $\mathcal{D}_{T}^{+}$, and that $\partial_{t} h=0$ on $\Omega \times(0, T) \backslash\left(\mathcal{D}_{T}^{+} \cup \mathcal{D}_{T}^{-}\right)$, we get

$$
\begin{gathered}
\int_{\Omega} \int_{0}^{T}\left(w_{i}(x, 0, t) d_{i}^{s}(x, t)+v_{i}(x, 0, t) q_{i}^{s}(x, t)-d_{i}^{s}(x, t) q_{i}^{s}(x, t)\right) \partial_{t} h(x, t) \mathrm{d} t \mathrm{~d} x \\
=\int_{\Omega} \int_{0}^{T} v_{i}(x, 0, t) w_{i}(x, 0, t) \partial_{t} h(x, t) \mathrm{d} t \mathrm{~d} x=0 .
\end{gathered}
$$

Equation (4.15) together with equation (4.12) conclude the proof of Theorem 2.3.

\section{Existence of A SOlution to the ADJOINT EQUATiOns}

The objective of this section is to prove Lemma 4.2 stating the existence of a weak solution to the adjoint problem (4.9). This proof will use the convergence of a finite volume numerical scheme in a similar way as in [4]. Thus, to fit into the framework of [4], we rather consider here the direct problem $(2.6)-(2.7)$ on $\Omega \times \mathbb{R}_{+}^{*} \times(0, T)$, $T>0$, but with non vanishing right hand sides $f_{i} \in \mathrm{L}^{\infty}\left(\Omega \times \mathbb{R}_{+}^{*} \times(0, T)\right)$ in the advection equations: using the same notations as previously, we study in this section the system

$$
\left\{\begin{array}{rlrl}
\left.u_{i}\right|_{\xi=0} \partial_{t} h+\operatorname{div}\left(-c_{i}^{s} \nabla h\right)=0 & & \text { on } \Omega \times(0, T), \\
\left.c_{i}^{s}\right|_{\Sigma_{T}^{+}}=\tilde{c}_{i} & & \text { on } \Sigma_{T}^{+} \\
\mathcal{L} u_{i} & =f_{i} & & \text { on } \Omega \times \mathbb{R}_{+}^{*} \times(0, T), \\
\left.u_{i}\right|_{\xi=0} & =c_{i}^{s} & & \text { on } \mathcal{D}_{T}^{+} \\
\left.u_{i}\right|_{t=0} & =u_{i}^{0} & & \text { on } \Omega \times \mathbb{R}_{+}^{*}
\end{array}\right.
$$

for all $i=1, \ldots, L$, with $h$ given by (2.6). Furthermore, no assumptions are made on the sign nor the sum over the lithologies of the boundary and initial conditions $\tilde{c}_{i}, u_{i}^{0}$, and in the sequel, the hypothesis made on the data are the following ones:

\section{Hypothesis 5.1.}

(i) $\Omega$ is an open bounded subset of $\mathbb{R}^{d}$, of class $\mathrm{C}^{\infty}$;

(ii) $h^{0} \in \mathrm{C}^{2}(\bar{\Omega})$;

(iii) $g \in \mathrm{C}^{1}\left(\partial \Omega \times \mathbb{R}_{+}^{*}\right) \cap \mathrm{L}^{2}\left(\partial \Omega \times \mathbb{R}_{+}^{*}\right)$;

(iv) $g$ and $h^{0}$ are chosen according to the assumptions of Theorem 5.3 of [11] (p. 320) so that the unique solution $h$ of $(2.6)$ is in $\mathrm{C}^{2}(\bar{\Omega} \times[0, T])$;

(v) $\tilde{c}_{i} \in \mathrm{L}^{\infty}\left(\Sigma_{2 T}^{+}\right)$for all $i=1, \ldots, L$;

(vi) $u_{i}^{0} \in \mathrm{L}^{\infty}\left(\Omega \times \mathbb{R}_{+}^{*}\right)$ for all $i=1, \ldots, L$;

(vii) $f_{i} \in \mathrm{L}^{\infty}\left(\Omega \times \mathbb{R}_{+}^{*} \times(0,2 T)\right)$ for all $i=1, \ldots, L$.

Since any weak solution of (2.6)-(2.7) is by definition in $\mathrm{L}^{\infty}$, the study of the adjoint problem (4.9) under Hypothesis 2.1 amounts to the study of the direct problem (5.1) under Hypothesis 5.1. 
To obtain a rigorous mathematical formulation of problem (5.1), we are looking for weak solutions defined as follows for all $i=1, \ldots, L$.

Definition 5.2. Let us assume that Hypothesis 5.1 holds, and let $h$ denote the solution of problem (2.6). Then $\left(u_{i}, c_{i}^{s}\right) \in \mathrm{L}^{\infty}\left(\Omega \times \mathbb{R}_{+}^{*} \times(0, T)\right) \times \mathrm{L}^{\infty}(\Omega \times(0, T))$ is said to be a weak solution of $(5.1)$ if it satisfies:

(i) for all $\varphi \in\left\{\phi \in \mathrm{C}_{c}^{\infty}\left(\mathbb{R}^{d+2}\right) \mid \phi(., 0,)=\right.$.0 on $\Omega \times(0, T) \backslash \mathcal{D}_{T}^{+}$and $\phi(., ., T)=0$ on $\left.\Omega \times \mathbb{R}_{+}\right\}$

$$
\begin{gathered}
\int_{\Omega} \int_{\mathbb{R}_{+}} \int_{0}^{T}(\mathcal{L} \varphi)(x, \xi, t) u_{i}(x, \xi, t) \mathrm{d} t \mathrm{~d} \xi \mathrm{d} x+\int_{\Omega} \int_{\mathbb{R}_{+}} \int_{0}^{T} f_{i}(x, \xi, t) \varphi(x, \xi, t) \mathrm{d} t \mathrm{~d} \xi \mathrm{d} x \\
\quad+\int_{\Omega} \int_{\mathbb{R}_{+}} u_{i}^{0}(x, \xi) \varphi(x, \xi, 0) \mathrm{d} \xi \mathrm{d} x+\int_{\Omega} \int_{0}^{T} \partial_{t} h(x, t) c_{i}^{s}(x, t) \varphi(x, 0, t) \mathrm{d} t \mathrm{~d} x=0,
\end{gathered}
$$

(ii) for all $\psi \in\left\{\phi \in \mathrm{C}_{c}^{\infty}\left(\mathbb{R}^{d+2}\right) \mid \phi(., 0,)=\right.$.0 on $\partial \Omega \times(0, T) \backslash \Sigma_{T}^{+}$and $\phi(., ., T)=0$ on $\left.\Omega \times \mathbb{R}_{+}\right\}$

$$
\begin{gathered}
-\int_{\Omega} \int_{\mathbb{R}_{+}} \int_{0}^{T}(\mathcal{L} \psi)(x, \xi, t) u_{i}(x, \xi, t) \mathrm{d} t \mathrm{~d} \xi \mathrm{d} x \\
-\int_{\Omega} \int_{\mathbb{R}_{+}} \int_{0}^{T} f_{i}(x, \xi, t) \psi(x, \xi, t) \mathrm{d} t \mathrm{~d} \xi \mathrm{d} x-\int_{\Omega} \int_{\mathbb{R}_{+}} u_{i}^{0}(x, \xi) \psi(x, \xi, 0) \mathrm{d} \xi \mathrm{d} x \\
+\int_{0}^{T}\left(\int_{\Omega} c_{i}^{s}(x, t) \nabla h(x, t) \cdot \nabla \psi(x, 0, t) \mathrm{d} x-\int_{\partial \Omega} \tilde{c}_{i}(x, t) g(x, t) \psi(x, 0, t) \mathrm{d} \gamma(x)\right) \mathrm{d} t=0 .
\end{gathered}
$$

In the following, we shall denote by $\bar{f}_{i}$ the function obtained by the change of variables $(x, z, t)=\left(x^{\prime}, h\left(x^{\prime}, t^{\prime}\right)-\right.$ $\left.z, t^{\prime}\right)$ in $f_{i}$ for all $i=1, \ldots, L$ :

$$
\bar{f}_{i}(x, z, t)=f_{i}(x, h(x, t)-z, t) \text { on } \mathcal{B}_{T}=\{(x, z, t) \mid(x, t) \in \Omega \times(0, T), z<h(x, t)\} .
$$

In this new coordinate system, the variables $c_{i}$ satisfy for all $i=1, \ldots, L$

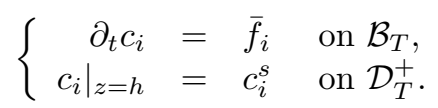

The aim of this section is to prove Theorem 5.3 stated below, which gives the existence of a weak solution to problem (5.1) in the sense of Definition 5.2. Under Hypothesis 2.1, Lemma 4.2 is a straightforward corollary of Theorem 5.3.

The proof of Theorem 5.3 is achieved by adapting the proof of convergence of the finite volume scheme (3.2)(3.5) given in [4] to the case of non vanishing right hand sides. It outlines as follows: first, the numerical scheme derived from (2.6) and (5.1) is given in Section 5.1. Then, the existence, uniqueness and stability of the discrete solutions are obtained (see Sect. 5.2), followed by the proof of convergence of these solutions towards a weak solution in the sense of Definition 5.2. In this last subsection, only the main differences with the proof given in [4] will be detailed.

\subsection{Finite volume scheme}

The finite volume scheme derived here is the same as the one given in Section 3, except for the column concentrations. Indeed, following equation (5.4) and using the same notation as in Section 3, the discrete unknown $c_{i, \kappa}^{n+1}(z), n \geq 0$, is here defined as the exact solution at time $t^{n+1}$ of the problem

$$
\left\{\begin{aligned}
\partial_{t} c_{i, \kappa}(z, t) & =\bar{f}_{i, \kappa}(z, t), & & \\
c_{i, \kappa}\left(z, t^{n}\right) & =c_{i, \kappa}^{n}(z) & & \text { if } z<h_{\kappa}^{n} \\
c_{i, \kappa}\left(h_{\kappa}(t), t\right) & =c_{i, n+1}^{s, n+1} & & \text { if } \partial_{t} h_{\kappa}(t)>0
\end{aligned}\right.
$$


for all $\kappa \in \mathcal{K}, t \in\left(t^{n}, t^{n+1}\right], z<h_{\kappa}(t)$, with

$$
\begin{gathered}
\bar{f}_{i, \kappa}(z, t)=\frac{1}{|\kappa|} \int_{\kappa} \bar{f}_{i}(x, z, t) \mathrm{d} x, \\
h_{\kappa}(t)=h_{\kappa}^{n}+\left(t-t^{n}\right) \partial_{t} h_{\kappa}(t), \quad \text { and } \partial_{t} h_{\kappa}(t)=\frac{h_{\kappa}^{n+1}-h_{\kappa}^{n}}{\Delta t} \text { for all } t \in\left(t^{n}, t^{n+1}\right] .
\end{gathered}
$$

This leads to the following discretization of equations (2.6) and (5.1):

Sediment thickness:

$$
|\kappa| \frac{h_{\kappa}^{n+1}-h_{\kappa}^{n}}{\Delta t}+\sum_{\kappa^{\prime} \in \mathcal{K}_{\kappa}} T_{\kappa \kappa^{\prime}}\left(h_{\kappa}^{n+1}-h_{\kappa^{\prime}}^{n+1}\right)-|\partial \kappa \cap \partial \Omega| g_{\kappa}^{n+1}=0
$$

Conservation of surface sediments:

$$
\frac{\Delta \mathcal{M}_{i, \kappa}^{n+1}}{\Delta t}|\kappa|+\sum_{\kappa^{\prime} \in \mathcal{K}_{\kappa}} c_{i, \kappa \kappa^{\prime}}^{s, n+1} T_{\kappa \kappa^{\prime}}\left(h_{\kappa}^{n+1}-h_{\kappa^{\prime}}^{n+1}\right)-|\partial \kappa \cap \partial \Omega| \tilde{c}_{i, \kappa}^{n+1} g_{\kappa}^{(+), n+1}+|\partial \kappa \cap \partial \Omega| c_{i, \kappa}^{s, n+1} g_{\kappa}^{(-), n+1}=0
$$

Column sediments:

$$
\begin{aligned}
& \Delta \mathcal{M}_{i, \kappa}^{n+1}= \begin{cases}c_{i, \kappa}^{s, n+1}\left(h_{\kappa}^{n+1}-h_{\kappa}^{n}\right) & \text { if } h_{\kappa}^{n+1} \geq h_{\kappa}^{n}, \\
\int_{h_{\kappa}^{n}}^{h_{\kappa}^{n+1}} c_{i, \kappa}^{n}(z) \mathrm{d} z & \text { otherwise }\end{cases} \\
& c_{i, \kappa}^{n+1}(z)= \begin{cases}c_{i, \kappa}^{n}(z)+\int_{t^{n}}^{t^{n+1}} \bar{f}_{i, \kappa}(z, t) \mathrm{d} t & \text { if } z \leq \min \left(h_{\kappa}^{n}, h_{\kappa}^{n+1}\right), \\
c_{i, \kappa}^{s, n+1}+\int_{t^{n}+\Delta t}^{t^{n+1}}\left(\frac{z-h_{\kappa}^{n}}{h_{\kappa}^{n+1}-h_{\kappa}^{n}}\right) \bar{f}_{i, \kappa}(z, t) \mathrm{d} t & \text { if } h_{\kappa}^{n}<z<h_{\kappa}^{n+1},\end{cases} \\
& u_{i, \kappa}^{n}(\xi)=c_{i, \kappa}^{n}\left(h_{\kappa}^{n}-\xi\right) \text { for all } \xi \in \mathbb{R}_{+}^{*} \text {. }
\end{aligned}
$$

In the next subsection, we shall prove the existence of solutions $\left(h_{\kappa}^{n}\right)_{\kappa \in \mathcal{K}},\left(c_{i, \kappa}^{s, n+1}\right)_{\kappa \in \mathcal{K}},\left(c_{i, \kappa}^{n}\right)_{\kappa \in \mathcal{K}}$ and $\left(u_{i, \kappa}^{n}\right)_{\kappa \in \mathcal{K}}$, $i=1, \ldots, L, n \in\left\{0, \ldots, N_{\Delta t}\right\}$, to problem (5.7)-(5.11). These solutions are unique except for the surface concentration $c_{i, \kappa}^{s, n+1}$ which is arbitrary at some degenerate points $(\kappa, n+1)$ for which it is chosen according to Lemma 5.4 stated below. For any admissible mesh $\left(\mathcal{K}, \Sigma_{\text {int }}, \mathcal{P}\right)$ of $\Omega$ in the sense of Definition 3.1 , any time step $\Delta t>0$, and $i=1, \ldots, L$, let us define, as in [4], the functions $h_{\mathcal{K}, \Delta t}, c_{i, \mathcal{K}, \Delta t}^{s}$ on $\Omega \times\left(0,\left(N_{\Delta t}+1\right) \Delta t\right]$, and $u_{i, \mathcal{K}, \Delta t}$ on $\Omega \times \mathbb{R}_{+}^{*} \times\left(0,\left(N_{\Delta t}+1\right) \Delta t\right]$, by

$$
\left\{\begin{aligned}
h_{\mathcal{K}, \Delta t}(x, t) & =h_{\kappa}^{n+1} \\
u_{i, \mathcal{K}, \Delta t}(x, \xi, t) & =u_{i, \kappa}^{n+1}(\xi) \\
c_{i, \mathcal{K}, \Delta t}^{s}(x, t) & =c_{i, \kappa}^{s, n+1}
\end{aligned}\right.
$$

for all $x \in \kappa, \kappa \in \mathcal{K}, t \in\left(t^{n}, t^{n+1}\right], n \in\left\{0, \ldots, N_{\Delta t}\right\}, \xi \in \mathbb{R}_{+}^{*}$, where $h_{\kappa}^{n}, c_{i, \kappa}^{s, n+1}$, and $u_{i, \kappa}^{n}$ are any given solution of (5.7)-(5.11) chosen according to Lemma 5.4.

Then, the objective of this section is to prove the following theorem:

Theorem 5.3. Hypothesis 5.1 is assumed to hold. For all $m \in \mathbb{N}$, let $\left(\mathcal{K}_{m}, \Sigma_{\text {int }}^{m}, \mathcal{P}_{m}\right)$ be an admissible mesh of $\Omega$ in the sense of Definition 3.1 and $\Delta t_{m} \in(0, T)$. Let us assume that there exists $\alpha>0$ such that reg $\left(\mathcal{K}_{m}\right) \leq \alpha$ for all $m \in \mathbb{N}$, and that $\Delta t_{m} \rightarrow 0, \frac{\delta \mathcal{K}_{m}}{\sqrt{\Delta t_{m}}} \rightarrow 0$ as $m \rightarrow \infty$.

For all $m \in \mathbb{N}$ and $i=1, \ldots, L$, let $h_{\mathcal{K}_{m}, \Delta t_{m}}, u_{i, \mathcal{K}_{m}, \Delta t_{m}}$ denote the unique functions defined by (5.12), and $c_{i, \mathcal{K}_{m}, \Delta t_{m}}$ be a function defined by (5.12) from any solution of (5.7)-(5.11) chosen according to Lemma 5.4 with $\mathcal{K}=\mathcal{K}_{m}, \Delta t=\Delta t_{m}$ 
Then, the sequence $\left(h_{\mathcal{K}_{m}, \Delta t_{m}}\right)_{m \in \mathbb{N}}$ converges to the solution $h$ of problem $(2.6)$ in $\mathrm{L}^{\infty}\left(0, T ; \mathrm{L}^{2}(\Omega)\right)$, and there exists a subsequence of $\left(\mathcal{K}_{m}, \Delta t_{m}\right)_{m \in \mathbb{N}}$, still denoted by $\left(\mathcal{K}_{m}, \Delta t_{m}\right)_{m \in \mathbb{N}}$, such that, for all $i \in\{1, \ldots, L\}$, the subsequence $\left(c_{i, \mathcal{K}_{m}, \Delta t_{m}}\right)_{m \in \mathbb{N}}$ (resp. $\left.\left(u_{i, \mathcal{K}_{m}, \Delta t_{m}}\right)_{m \in \mathbb{N}}\right)$ converges to a function $c_{i}^{s}$ in $\mathrm{L}^{\infty}(\Omega \times(0, T))\left(\right.$ resp. $u_{i}$ in

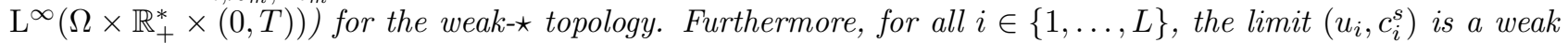
solution of problem (5.1) in the sense of Definition 5.2.

\subsection{Proof of Theorem 5.3}

The proof of Theorem 5.3 is very similar to the one giving the existence of a weak solution to problem (2.7) in the sense of Definition 2.2 and developed in [4].

The existence, uniqueness and convergence of the sequence of discrete sediment thicknesses $\left(h_{\mathcal{K}_{m}, \Delta t_{m}}\right)_{m \in \mathbb{N}}$ to the solution of (2.6) have already been shown in [4], as well as the following bounds, used in the sequel: for any $m \in \mathbb{N}$,

$$
\begin{gathered}
\sum_{n=0}^{N_{\Delta t_{m}}+1} \Delta t_{m} \sum_{\kappa \in \mathcal{K}_{m}}|\kappa|\left(\frac{h_{\kappa}^{n+1}-h_{\kappa}^{n}}{\Delta t_{m}}\right)^{2} \leq D_{1}, \\
\left\|h-h_{\mathcal{K}_{m}, \Delta t_{m}}\right\|_{\mathrm{L}^{\infty}\left(0, T ; \mathrm{L}^{2}(\Omega)\right)} \leq D_{2}\left(\delta \mathcal{K}_{m}+\Delta t_{m}\right),
\end{gathered}
$$

with $D_{1}$ (resp. $\left.D_{2}\right)$ only depending on $h, \Omega, T$ and $\alpha$ (resp. on $h$ and $\Omega$ ).

Concerning the concentration variables, the system (3.2)-(3.5) differs from (5.7)-(5.11) by the right hand sides $f_{i}$ in $\mathrm{L}^{\infty}\left(\Omega \times \mathbb{R}_{+}^{*} \times(0,2 T)\right), i=1, \ldots, L$, in the advection equations and by the unconstrained values in $\mathrm{L}^{\infty}$ of the initial and boundary conditions. Despite these differences, the same stages as in [4] are followed to prove the existence of a weak solution to the coupled problem: we first show the existence of a bounded solution for the discrete concentrations (Lem. 5.4), which yields the convergence of these concentrations in $\mathrm{L}^{\infty}$ for the weak- $\star$ topology (Prop. 5.5). Then, a linear advection equation satisfied in the weak sense by the discrete solutions is obtained (Prop. 5.6), and is finally used to show the existence of a weak solution.

Lemma 5.4. Under Hypothesis 5.1 , let $\left(\mathcal{K}, \Sigma_{\text {int }}, \mathcal{P}\right)$ be an admissible mesh of $\Omega$ in the sense of Definition 3.1 , $\Delta t \in(0, T), M_{i}=\max \left(\left\|u_{i}^{0}\right\|_{\mathrm{L}^{\infty}\left(\Omega \times \mathbb{R}_{+}^{*}\right)},\left\|\tilde{c}_{i}\right\|_{\mathrm{L}^{\infty}\left(\Sigma_{2 T}^{+}\right)}\right)+2 T\left\|f_{i}\right\|_{\mathrm{L}^{\infty}\left(\Omega \times \mathbb{R}_{+}^{*} \times(0,2 T)\right)}$ for all $i \in\{1, \ldots, L\}$ and, for all $n \in\left\{0, \ldots, N_{\Delta t}+1\right\}$, let $\left(h_{\kappa}^{n}\right)_{\kappa \in \mathcal{K}}$ be the solution of $(5.7)$. For $i \in\{1, \ldots, L\}$ and $n \in\left\{0, \ldots, N_{\Delta t}\right\}$, there exists a unique solution $\left(c_{i, \kappa}^{n+1}\right)_{\kappa \in \mathcal{K}}$, and there exists at least one solution $\left(c_{i, \kappa}^{s, n+1}\right)_{\kappa \in \mathcal{K}}$ to the set of equations (5.8)-(5.11) such that

$$
\left|c_{i, \kappa}^{s, n+1}\right| \leq M_{i} \text { for all } \kappa \in \mathcal{K} \text { and } n \in\left\{0, \ldots, N_{\Delta t}\right\}
$$

Furthermore, one has

$$
\left|c_{i, \kappa}^{n}(z)\right| \leq M_{i} \text { for all } \kappa \in \mathcal{K}, n \in\left\{0, \ldots, N_{\Delta t}+1\right\} \text { and } z<h_{\kappa}^{n}
$$

Proof. Since the discrete accumulation term is the same as in problem (3.2)-(3.5), the proof of existence and uniqueness of the approximate concentrations $\left(c_{i, \kappa}^{s, n+1}\right)_{\kappa \in \mathcal{K}},\left(c_{i, \kappa}^{n}\right)_{\kappa \in \mathcal{K}}$ and $\left(u_{i, \kappa}^{n}\right)_{\kappa \in \mathcal{K}}, i=1, \ldots, L$, is unchanged (see [4] for details).

The inequalities (5.15) and (5.16) are proved by induction over $n \in\left\{0, \ldots, N_{\Delta t}\right\}$ and over the control volumes in decreasing topographical order. Let us consider a control volume $\kappa \in \mathcal{K}$ and a time step $n \geq 0$. The induction hypothesis assumes that $\left|c_{i, \kappa^{\prime}}^{n}(z)\right| \leq M_{i}^{n}$ for all $\kappa^{\prime} \in \mathcal{K}$, and that, for the higher cells $\kappa^{\prime} \in \mathcal{K}$ such that $h_{\kappa}^{n+1}<h_{\kappa^{\prime}}^{n+1},\left|c_{i, \kappa^{\prime}}^{s, n+1}\right| \leq M_{i}^{n}$, with $M_{i}^{n}=\max \left(\left\|u_{i}^{0}\right\|_{L^{\infty}\left(\Omega \times \mathbb{R}_{+}^{*}\right)},\left\|\tilde{c_{i}}\right\|_{L^{\infty}\left(\Sigma_{2 T}^{+}\right)}\right)+n \Delta t\left\|f_{i}\right\|_{L^{\infty}\left(\Omega \times \mathbb{R}_{+}^{*} \times(0,2 T)\right)}$. 
Let us first consider the erosion case for which $h_{\kappa}^{n+1} \leq h_{\kappa}^{n}$. It results from the induction hypothesis over $n$ and over the control volumes that

$$
\begin{gathered}
\left|c_{i, \kappa}^{s, n+1}\right|\left(\sum_{\substack{\kappa^{\prime} \in \mathcal{K}_{\kappa} \\
h_{\kappa}>h_{\kappa^{\prime}}}} T_{\kappa \kappa^{\prime}}\left(h_{\kappa}^{n+1}-h_{\kappa^{\prime}}^{n+1}\right)+|\partial \kappa \cap \partial \Omega| g_{\kappa}^{(-), n+1}\right) \\
\leq M_{i}^{n}\left(|\partial \kappa \cap \partial \Omega| g_{\kappa}^{(+), n+1}+\sum_{\substack{\kappa^{\prime} \in \mathcal{K}_{\kappa} \\
h_{\kappa} \leq h_{\kappa^{\prime}}}} T_{\kappa \kappa^{\prime}}\left(h_{\kappa^{\prime}}^{n+1}-h_{\kappa}^{n+1}\right)+\frac{|\kappa|}{\Delta t}\left(h_{\kappa}^{n}-h_{\kappa}^{n+1}\right)\right) .
\end{gathered}
$$

Thus, using the fact that the discrete sediment thickness satisfies equation (5.7), we get

$$
\left(\left|c_{i, \kappa}^{s, n+1}\right|-M_{i}^{n}\right) \underbrace{\left(\sum_{\substack{\kappa^{\prime} \in \mathcal{K}_{\kappa} \\ h_{\kappa}>h_{\kappa^{\prime}}}} T_{\kappa \kappa^{\prime}}\left(h_{\kappa}^{n+1}-h_{\kappa^{\prime}}^{n+1}\right)+|\partial \kappa \cap \partial \Omega| g_{\kappa}^{(-), n+1)}\right)}_{A_{\kappa}^{+}} \leq 0 .
$$

In this equation, either the term $A_{\kappa}^{+}$is strictly positive, or it vanishes. In the first case, it results that $\left|c_{i, \kappa}^{s, n+1}\right| \leq M_{i}^{n}$ for all $i=1, \ldots, L$. In the second case, the point $(\kappa, n+1)$ is said to be degenerated in the sense that the concentrations $c_{i, \kappa}^{s, n+1}$ can be chosen arbitrarily in the interval $\left[-M_{i}^{n}, M_{i}^{n}\right]$.

Let us now assume that $h_{\kappa}^{n+1}>h_{\kappa}^{n}$ (sedimentation). Proceeding as above, we get

$$
\begin{gathered}
\left|c_{i, \kappa}^{s, n+1}\right|\left(\frac{|\kappa|}{\Delta t}\left(h_{\kappa}^{n+1}-h_{\kappa}^{n}\right)+\sum_{\substack{\kappa^{\prime} \in \mathcal{K}_{\kappa} \\
h_{\kappa}>h_{\kappa^{\prime}}}} T_{\kappa \kappa^{\prime}}\left(h_{\kappa}^{n+1}-h_{\kappa^{\prime}}^{n+1}\right)+|\partial \kappa \cap \partial \Omega| g_{\kappa}^{(-), n+1}\right) \\
\leq M_{i}^{n}\left(|\partial \kappa \cap \partial \Omega| g_{\kappa}^{(+), n+1}+\sum_{\substack{\kappa^{\prime} \in \mathcal{K}_{\kappa} \\
h_{\kappa} \leq h_{\kappa^{\prime}}}} T_{\kappa \kappa^{\prime}}\left(h_{\kappa^{\prime}}^{n+1}-h_{\kappa}^{n+1}\right)\right)
\end{gathered}
$$

which yields, according to equation (5.7),

$$
\left(\left|c_{i, \kappa}^{s, n+1}\right|-M_{i}^{n}\right)\left(\frac{|\kappa|}{\Delta t}\left(h_{\kappa}^{n+1}-h_{\kappa}^{n}\right)+\sum_{\substack{\kappa^{\prime} \in \mathcal{K}_{\kappa} \\ h_{\kappa}>h_{\kappa^{\prime}}}} T_{\kappa \kappa^{\prime}}\left(h_{\kappa}^{n+1}-h_{\kappa^{\prime}}^{n+1}\right)+|\partial \kappa \cap \partial \Omega| g_{\kappa}^{(-), n+1}\right) \leq 0 .
$$

Since we have assumed $h_{\kappa}^{n+1}>h_{\kappa}^{n}$, the second term into brackets is strictly positive, and thus $\left|c_{i, \kappa}^{s, n+1}\right| \leq M_{i}^{n}$.

This proof is still valid for $n=0$ and for all the highest cells $\kappa_{0} \in \mathcal{K}$ at any time $t^{n+1}, n \geq 0$.

Finally, concerning the basin concentrations, we have by definition $\left|c_{i, \kappa}^{0}(z)\right| \leq\left\|u_{i}^{0}\right\|_{L^{\infty}\left(\Omega \times \mathbb{R}_{+}^{*}\right)}$ and, for $n \in$ $\left\{0, \ldots, N_{\Delta t}\right\},(5.10)$ easily gives the inequality

$$
\left\|c_{i, \kappa}^{n+1}(.)\right\|_{\mathrm{L}^{\infty}\left(-\infty, h_{\kappa}^{n+1}\right)} \leq \max \left(\left\|c_{i, \kappa}^{n}(.)\right\|_{\mathrm{L}^{\infty}\left(-\infty, h_{\kappa}^{n}\right)},\left|c_{i, \kappa}^{s, n+1}\right|\right)+\Delta t\left\|f_{i}\right\|_{\mathrm{L}^{\infty}\left(\Omega \times \mathbb{R}_{+}^{*} \times(0,2 T)\right)} \leq M_{i}^{n+1},
$$

which concludes the proof. 
Denoting by $c_{i, \kappa}(z, t)$ the exact solution at time $t$ of (5.5) for all $\kappa \in \mathcal{K}, t \in\left(0,\left(N_{\Delta t}+1\right) \Delta t\right]$ and $z<h_{\kappa}(t)$, we can extend the discrete solutions $\left(u_{i, \kappa}^{n}\right)_{n \in\left\{0, \ldots, N_{\Delta t}+1\right\}}$ and $\left(c_{i, \kappa}^{s, n+1}\right)_{n \in\left\{0, \ldots, N_{\Delta t}\right\}}$ given by Lemma 5.4 to $t \in$ $\left(0,\left(N_{\Delta t}+1\right) \Delta t\right]$ for all $\kappa \in \mathcal{K}$ as follows:

$$
\begin{gathered}
u_{i, \kappa}(\xi, t)=c_{i, \kappa}\left(h_{\kappa}(t)-\xi, t\right) \text { for all } t \in\left(0,\left(N_{\Delta t}+1\right) \Delta t\right] \text { and } \xi \in \mathbb{R}_{+}^{*}, \\
\qquad c_{i, \kappa}^{s}(t)=c_{i, \kappa}^{s, n+1} \text { for all } t \in\left(t^{n}, t^{n+1}\right] .
\end{gathered}
$$

And we can easily deduce from Lemma 5.4 that the functions $c_{i, \kappa}(z, t)$ and $u_{i, \kappa}(\xi, t)$ are bounded in the interval $\left[-M_{i}, M_{i}\right]$ for all $i=1, \ldots, L$.

Let us now define $\bar{u}_{i, \mathcal{K}, \Delta t}$ on $\Omega \times \mathbb{R}_{+}^{*} \times\left(0,\left(N_{\Delta t}+1\right) \Delta t\right)$ by

$$
\bar{u}_{i, \mathcal{K}, \Delta t}(x, \xi, t)=u_{i, \kappa}(\xi, t)
$$

for all $x \in \kappa, \kappa \in \mathcal{K}$. Then we have the following proposition:

Proposition 5.5. For all $m \in \mathbb{N}$, let $\left(\mathcal{K}_{m}, \Sigma_{\text {int }}^{m}, \mathcal{P}_{m}\right)$ be an admissible mesh of $\Omega$ in the sense of Definition 3.1 and $\Delta t_{m} \in(0, T)$. Let us assume that $\Delta t_{m} \rightarrow 0$ and $\delta \mathcal{K}_{m} \rightarrow 0$ as $m \rightarrow \infty$.

For all $m \in \mathbb{N}$ and $i=1, \ldots, L$, let $u_{i, \mathcal{K}_{m}, \Delta t_{m}}$ (resp. $\bar{u}_{i, \mathcal{K}_{m}, \Delta t_{m}}$ ) denote the unique function defined by (5.12) (resp. by (5.19)) and $c_{i, \mathcal{K}_{m}, \Delta t_{m}}$ be a function defined by (5.12), from any solution of (5.8)-(5.11) chosen according to Lemma 5.4 with $\mathcal{K}=\mathcal{K}_{m}, \Delta t=\Delta t_{m}$.

Then, under Hypothesis 5.1, there exists a subsequence of $\left(\mathcal{K}_{m}, \Delta t_{m}\right)_{m \in \mathbb{N}}$, still denoted by $\left(\mathcal{K}_{m}, \Delta t_{m}\right)_{m \in \mathbb{N}}$, such that for all $i \in\{1, \ldots, L\}$

(i) the subsequence $\left(c_{i, \mathcal{K}_{m}, \Delta t_{m}}^{s}\right)_{m \in \mathbb{N}}$ converges to a function $c_{i}^{s}$ in $\mathrm{L}^{\infty}(\Omega \times(0, T))$ for the weak-太 topology,

(ii) the subsequences $\left(u_{i, \mathcal{K}_{m}, \Delta t_{m}}\right)_{m \in \mathbb{N}}$ and $\left(\bar{u}_{i, \mathcal{K}_{m}, \Delta t_{m}}\right)_{m \in \mathbb{N}}$ converge to a function $u_{i}$ in $\mathrm{L}^{\infty}\left(\Omega \times \mathbb{R}_{+}^{*} \times(0, T)\right)$ for the weak-太 topology.

Proof. Denoting by $u_{i}\left(\right.$ resp. $\left.\bar{u}_{i}\right)$ the limit as $m \rightarrow \infty$ of the subsequence $\left(u_{i, \mathcal{K}_{m}, \Delta t_{m}}\right)_{m \in \mathbb{N}}\left(\right.$ resp. $\left.\left(\bar{u}_{i, \mathcal{K}_{m}, \Delta t_{m}}\right)_{m \in \mathbb{N}}\right)$ in $\mathrm{L}^{\infty}\left(\Omega \times \mathbb{R}_{+}^{*} \times(0, T)\right)$, the only difficulty is to prove that $u_{i}=\bar{u}_{i}$. It is achieved as in the proof of Proposition 5.2 in [4] using the assumption $f_{i} \in \mathrm{L}^{\infty}\left(\Omega \times \mathbb{R}_{+}^{*} \times(0,2 T)\right)$, the bounds (5.13), (5.15), (5.16) on the solutions, and the following relation: for $x \in \kappa, \kappa \in \mathcal{K}_{m}$ and $t \in\left(t^{n}, t^{n+1}\right]$,

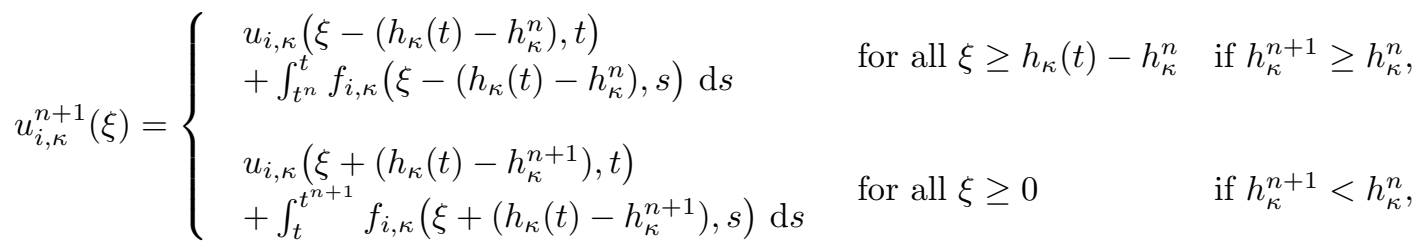

with $f_{i, \kappa}(\xi, t)=\bar{f}_{i, \kappa}\left(h_{\kappa}(t)-\xi, t\right)$.

Then, to show the convergence of the approximate solutions towards a weak solution of the coupled problem, we state, as in [4], that the functions $c_{i, \kappa}(z, t)$ satisfy a linear advection equation. Indeed, using equation (5.5), Lemma 5.5 of [4] extends to:

Proposition 5.6. Let us assume that Hypothesis 5.1 holds and let $h$ denote the solution of problem (2.6). Let $\left(\mathcal{K}, \Sigma_{\text {int }}, \mathcal{P}\right)$ be an admissible mesh of $\Omega$ in the sense of Definition 3.1, and $\Delta t \in(0, T)$.

Let $h_{\mathcal{K}, \Delta t}, u_{i, \mathcal{K}, \Delta t}, i=1, \ldots, L$, (resp. $\left.\bar{u}_{i, \mathcal{K}, \Delta t}, i=1, \ldots, L\right)$ denote the unique functions defined by (5.12) (resp. by (5.19)) and $c_{i, \mathcal{K}, \Delta t}^{s}, i=1, \ldots, L$, be a function defined by (5.12), from any solution of (5.8)-(5.11) chosen according to Lemma 5.4 . 
Then, for any $\kappa \in \mathcal{K}$ and $i \in\{1, \ldots, L\}$ :

(i) for all $\varphi \in\left\{\phi \in \mathrm{C}_{c}^{\infty}\left(\mathbb{R}^{2}\right) \mid \phi(., T)=0\right.$ on $\left.\mathbb{R}_{+}\right\}$,

$$
\begin{gathered}
\int_{0}^{T} \int_{\mathbb{R}_{+}}\left[\partial_{t} \varphi(\xi, t)+\partial_{t} h_{\kappa}(t) \partial_{\xi} \varphi(\xi, t)\right] u_{i, \kappa}(\xi, t) \mathrm{d} \xi \mathrm{d} t+\int_{0}^{T} \int_{\mathbb{R}_{+}} f_{i, \kappa}(\xi, t) \varphi(\xi, t) \mathrm{d} \xi \mathrm{d} t \\
+\int_{\mathbb{R}_{+}} u_{i, \kappa}^{0}(\xi) \varphi(\xi, 0) \mathrm{d} \xi+\int_{0}^{T} \partial_{t} h_{\kappa}(t) u_{i, \kappa}(0, t) \varphi(0, t) \mathrm{d} t=0
\end{gathered}
$$

(ii) for all $\varphi \in\left\{\phi \in \mathrm{C}_{c}^{\infty}\left(\mathbb{R}^{2}\right) \mid \phi(., T)=0\right.$ on $\mathbb{R}_{+}$and $\phi(0, t)=0$ for all $t>0$ such that $\left.\partial_{t} h_{\kappa}(t) \leq 0\right\}$,

$$
\begin{gathered}
\int_{0}^{T} \int_{\mathbb{R}_{+}}\left[\partial_{t} \varphi(\xi, t)+\partial_{t} h_{\kappa}(t) \partial_{\xi} \varphi(\xi, t)\right] u_{i, \kappa}(\xi, t) \mathrm{d} \xi \mathrm{d} t+\int_{0}^{T} \int_{\mathbb{R}_{+}} f_{i, \kappa}(\xi, t) \varphi(\xi, t) \mathrm{d} \xi \mathrm{d} t \\
+\int_{\mathbb{R}_{+}} u_{i, \kappa}^{0}(\xi) \varphi(\xi, 0) \mathrm{d} \xi+\int_{0}^{T} \partial_{t} h_{\kappa}(t) c_{i, \kappa}^{s}(t) \varphi(0, t) \mathrm{d} t=0 .
\end{gathered}
$$

Let us now prove Theorem 5.3.

The proof of convergence of the numerical scheme (3.2)-(3.5) in [4] does not directly use the value of the bounds on the discrete solutions nor the value of the sum over the lithologies of the discrete concentrations, but only the stability of the solutions and the decoupling of the sediment thickness variable from the concentration variables. Thus, to complete the proof in our case, we just need to show the convergence of the terms involving the functions $f_{i}, i=1, \ldots, L$.

The first expression (5.2) in the weak formulation is obtained using the discrete linear advection equation (5.20) applied to $\varphi\left(x_{\kappa}, \xi, t\right), \varphi \in\left\{\phi \in \mathrm{C}_{c}^{\infty}\left(\mathbb{R}^{d+2}\right) \mid \phi(., 0,)=\right.$.0 on $\Omega \times(0, T) \backslash \mathcal{D}_{T}^{+}$and $\phi(., ., T)=0$ on $\Omega \times$ $\left.\mathbb{R}_{+}\right\}$. The convergence of this equation towards (5.2) has already been shown in [4] in the case $f_{i}=0$. Thus, there only remains to prove that:

$$
A_{i, m}=\sum_{\kappa \in \mathcal{K}_{m}}|\kappa| \int_{0}^{T} \int_{\mathbb{R}_{+}} f_{i, \kappa}(\xi, t) \varphi\left(x_{\kappa}, \xi, t\right) \mathrm{d} \xi \mathrm{d} t \rightarrow \int_{\Omega} \int_{\mathbb{R}_{+}} \int_{0}^{T} f_{i}(x, \xi, t) \varphi(x, \xi, t) \mathrm{d} t \mathrm{~d} \xi \mathrm{d} x
$$

as $m \rightarrow \infty$.

Then, we will show the convergence towards (5.3) of the sum over $\kappa \in \mathcal{K}_{m}, n \in\left\{0, \ldots, N_{\Delta t_{m}}\right\}$ of equation (5.8) multiplied by $\varphi\left(x_{\kappa}, 0, t^{n+1}\right), \varphi \in\left\{\phi \in \mathrm{C}_{c}^{\infty}\left(\mathbb{R}^{d+2}\right) \mid \phi(., 0,)=\right.$.0 on $\partial \Omega \times(0, T) \backslash \Sigma_{T}^{+}$and $\phi(., ., T)=0$ on $\Omega \times$ $\left.\mathbb{R}_{+}\right\}$. Proceeding as in [4] and using (5.22), it amounts to prove that

$$
B_{i, m}=\sum_{\kappa \in \mathcal{K}_{m}}|\kappa| \int_{0}^{T} \partial_{t} h_{\kappa}(t) u_{i, \kappa}(0, t) \varphi\left(x_{\kappa}, 0, t\right) \mathrm{d} t-\sum_{\kappa \in \mathcal{K}_{m}}|\kappa| \sum_{n=0}^{N_{\Delta t_{m}}} \Delta \mathcal{M}_{i, \kappa}^{n+1} \varphi\left(x_{\kappa}, 0, t^{n+1}\right) \rightarrow 0
$$

as $m \rightarrow \infty$.

Let us now prove (5.22) and (5.23).

First, it can be shown that the function $f_{i, \mathcal{K}_{m}}$, defined by $f_{i, \mathcal{K}_{m}}(x, \xi, t)=f_{i, \kappa}(\xi, t)=\frac{1}{|\kappa|} \int_{\kappa} \bar{f}_{i}\left(y, h_{\kappa}(t)-\xi, t\right) \mathrm{d} y$ for all $x \in \kappa, \kappa \in \mathcal{K}_{m}, \xi \in \mathbb{R}_{+}^{*}, t \in(0, T)$, converges towards $f_{i}$ in $\mathrm{L}_{l o c}^{1}\left(\Omega \times \mathbb{R}_{+}^{*} \times(0, T)\right)$ using a density argument, the assumption $f_{i} \in \mathrm{L}^{\infty}\left(\Omega \times \mathbb{R}_{+}^{*} \times(0,2 T)\right)$, and the bounds (5.13), (5.14). This result readily implies (5.22).

Then, for any $\kappa \in \mathcal{K}_{m}, n \in\left\{0, \ldots, N_{\Delta t_{m}}\right\}$ and $i \in\{1, \ldots, L\}$, we get

$$
\Delta \mathcal{M}_{i, \kappa}^{n+1}=\int_{t^{n}}^{t^{n+1}} u_{i, \kappa}(0, t) \partial_{t} h_{\kappa}(t) \mathrm{d} t-\left\{\begin{array}{ll}
\int_{t^{n}}^{t^{n+1}} \\
0
\end{array}\left(\int_{t^{n}}^{t} \bar{f}_{i, \kappa}\left(h_{\kappa}(t), s\right) \mathrm{d} s\right) \partial_{t} h_{\kappa}(t) \mathrm{d} t \quad \begin{array}{l}
\text { if } h_{\kappa}^{n+1}<h_{\kappa}^{n} \\
\text { otherwise }
\end{array}\right.
$$


Substituting this equality in the definition of $B_{i, m}$ leads to

$$
\begin{aligned}
& B_{i, m}=\sum_{\kappa \in \mathcal{K}_{m}} \int_{\kappa} \int_{T}^{t^{N_{\Delta t_{m}}+1}} \bar{u}_{i, \mathcal{K}_{m}, \Delta t_{m}}(x, 0, t) \delta_{t} h_{\mathcal{K}_{m}, \Delta t_{m}}(x, t) \varphi\left(x_{\kappa}, 0, t\right) \mathrm{d} t \mathrm{~d} x \\
& +\sum_{\kappa \in \mathcal{K}_{m}} \sum_{n=0}^{N_{\Delta t_{m}}} \int_{\kappa} \int_{t^{n}}^{t^{n+1}} \bar{u}_{i, \mathcal{K}_{m}, \Delta t_{m}}(x, 0, t) \delta_{t} h_{\mathcal{K}_{m}, \Delta t_{m}}(x, t)\left[\varphi\left(x_{\kappa}, 0, t^{n+1}\right)-\varphi\left(x_{\kappa}, 0, t\right)\right] \mathrm{d} t \mathrm{~d} x \\
& -\sum_{n=0}^{N_{\Delta t m}} \int_{t^{n}}^{t^{n+1}} \sum_{\substack{\kappa \in \mathcal{K}_{m} \\
h_{\kappa}^{n+1}<h_{\kappa}^{n}}}|\kappa|\left(\int_{t^{n}}^{t} \bar{f}_{i, \kappa}\left(h_{\kappa}(t), s\right) \mathrm{d} s\right) \partial_{t} h_{\kappa}(t) \varphi\left(x_{\kappa}, 0, t^{n+1}\right) \mathrm{d} t,
\end{aligned}
$$

with $\delta_{t} h_{\mathcal{K}_{m}, \Delta t_{m}}(x, t)=\partial_{t} h_{\kappa}(t)=\left(h_{\kappa}^{n+1}-h_{\kappa}^{n}\right) / \Delta t_{m}$ for all $x \in \kappa, \kappa \in \mathcal{K}_{m}, t \in\left(t^{n}, t^{n+1}\right]$. Thanks to the regularity of $\varphi$, there exists $D_{3}>0$, only depending on $\varphi$, such that $\left|\varphi\left(x_{\kappa}, 0, t^{n+1}\right)-\varphi\left(x_{\kappa}, 0, t\right)\right| \leq D_{3} \Delta t_{m}$ for all $t \in\left[t^{n}, t^{n+1}\right]$. Since the function $\delta_{t} h_{\mathcal{K}_{m}, \Delta t_{m}}$ is uniformly bounded in $\mathrm{L}^{2}\left(\Omega \times\left(0, t^{N_{\Delta t_{m}}+1}\right)\right)$ (see (5.13)), since $\bar{u}_{i, \mathcal{K}_{m}, \Delta t_{m}} \in\left[-M_{i}, M_{i}\right], \bar{f}_{i} \in \mathrm{L}^{\infty}\left(\Omega \times \mathbb{R}_{+}^{*} \times(0,2 T)\right)$ and $\left|t^{N_{\Delta t_{m}}+1}-T\right|<\Delta t_{m}$, the convergence of $B_{i, m}$ to 0 as $m \rightarrow \infty$ is obtained: the limit $\left(u_{i}, c_{i}^{s}\right)$ satisfies equation (5.3), which ends the proof of Theorem 5.3.

\section{Proof of Lemma 4.1}

The proof of Lemma 4.1 uses the characteristics $\zeta(. ; x, \xi, t)$ and the so called "characteristic solution" $v_{c}$ of equation (4.1) (see [7]) defined below (see (6.4)) which is shown to be the unique weak solution of (4.1) and to satisfy the properties of Lemma 4.1 .

The proof uses a partition of unity on a covering of $\mathbb{R}^{d+2}$ built from the characteristics such that on each open set of the covering intersecting the boundary $\Omega \times \partial\left[\mathbb{R}_{+}^{*} \times(0, T)\right]$, the characteristic solution $v_{c}$ is regular assuming regular data. This will enable an easy derivation of the integration by part formulae stated in Lemma 4.1 . In order to stay away from the boundaries $\partial \mathcal{D}_{T}^{+} \times\{\xi=0\}, \partial \mathcal{D}_{T}^{-} \times\{\xi=0\}$, and $\Omega \times\{\xi=0\} \times\{t=0, T\}$, we shall need in addition a trick from [2] to prove that their contribution in the integration by part vanishes.

For all $(x, \xi, t) \in \bar{\Omega} \times \mathbb{R}_{+} \times \mathbb{R}_{+}$, let us define the characteristic $\zeta(. ; x, \xi, t)$ by

$$
\zeta(s ; x, \xi, t)=\int_{t}^{s} \partial_{t} h(x, r) \mathrm{d} r+\xi=h(x, s)-h(x, t)+\xi
$$

for all $s \in \mathbb{R}_{+}$. For all $(x, \xi, t) \in \bar{\Omega} \times \mathbb{R}_{+} \times[0, T]$, the input time $\tau_{e}$ and the output time $\tau_{s}$ of the characteristic $\zeta(. ; x, \xi, t)$ into and out of the domain $\Omega \times \mathbb{R}_{+}^{*} \times(0, T)$ are defined by

$$
\left\{\begin{array}{l}
\tau_{e}(x, \xi, t)=\inf \{s \text { such that } 0 \leq s \leq t \text { and } \zeta(s ; x, \xi, t) \geq 0\} \\
\tau_{s}(x, \xi, t)=\sup \{s \text { such that } t \leq s \leq T \text { and } \zeta(s ; x, \xi, t) \geq 0\}
\end{array}\right.
$$

We can prove the following lemma.

Lemma 6.1. For all $(x, \xi, t) \in \Omega \times \mathbb{R}_{+} \times[0, T]$ such that $\tau=\tau_{e}(x, \xi, t)>0$, then $(x, \tau) \in \overline{\mathcal{D}_{T}^{+}} \cap \Omega \times(0, T]$.

Let us now introduce the covering of $\mathbb{R}^{d+2}$ built from the characteristics such that on each open set, we can control the smoothness of the solutions of the direct and adjoint advection equations and derive the integration by part formulae.

From the regularity of the boundary $\partial \Omega$, it is possible to build a $\mathrm{C}^{2}$ extension of $h$ on an open neighbourhood $\omega \times[0, T]$ of $\bar{\Omega} \times[0, T]$ in $\mathbb{R}^{d} \times[0, T]$. Let us denote by $\bar{h}$ this extension and by $\bar{\zeta}$ the extension of $\zeta$ on $\omega \times \mathbb{R}_{+} \times[0, T]$. 
One can check that the set

$$
V_{0}=\left\{(x, \xi, \pm t) \mid x \in \omega, t \in[0, T), \xi=\bar{\zeta}(t ; x, \eta, 0) \text { with } \eta \in \mathbb{R}_{+}^{*}, \bar{\zeta}(s ; x, \eta, 0)>0 \text { for all } s \in[0, t]\right\}
$$

defines an open neighbourhood of $\bar{\Omega} \times \mathbb{R}_{+}^{*} \times\{t=0\}$. Similarly the set

$V_{T}=\left\{(x, \xi, t),(x, \xi, 2 T-t) \mid x \in \omega, t \in(0, T], \xi=\bar{\zeta}(t ; x, \eta, T)\right.$ with $\eta \in \mathbb{R}_{+}^{*}, \bar{\zeta}(s ; x, \eta, T)>0$ for all $\left.s \in[t, T]\right\}$

defines an open neighbourhood of $\bar{\Omega} \times \mathbb{R}_{+}^{*} \times\{t=T\}$.

Also, the set

$$
V_{\mathcal{D}^{+}}=\left\{(x, \pm \xi, t) \mid(x, t) \in \omega \times(0, T), \xi=\bar{\zeta}(t ; x, 0, s), t \geq s>0, \partial_{t} \bar{h}(x, r)>0 \text { for all } r \in[s, t]\right\}
$$

defines an open neighbourhood of $\left\{(x, t) \in \bar{\Omega} \times(0, T) \mid \partial_{t} h(x, t)>0\right\} \times\{\xi=0\}$, and the set

$$
V_{\mathcal{D}^{-}}=\left\{(x, \pm \xi, t) \mid(x, t) \in \omega \times(0, T), \xi=\bar{\zeta}(t ; x, 0, s), t \leq s<T, \partial_{t} \bar{h}(x, r)<0 \text { for all } r \in[t, s]\right\}
$$

defines an open neighbourhood of $\left\{(x, t) \in \bar{\Omega} \times(0, T) \mid \partial_{t} h(x, t)<0\right\} \times\{\xi=0\}$.

Let $\mathcal{D}^{0}$ denote the interior of the set $\left\{(x, t) \in \omega \times(0, T) \mid \partial_{t} \bar{h}(x, t)=0\right\}$ in $\omega \times(0, T)$, and consider the set

$$
V_{\mathcal{D}^{0}}=\left\{(x, \xi, t) \mid(x, t) \in \mathcal{D}^{0}, \xi \in(-1,1)\right\}
$$

which is an open neighbourhood of $\mathcal{D}^{0} \times\{\xi=0\}$.

Finally, in order to stay away from the set $S=B_{1} \cup B_{2} \cup B_{3} \cup B_{4}$ with $B_{1}=\partial \mathcal{D}_{T}^{+} \times\{\xi=0\}, B_{2}=\partial \mathcal{D}_{T}^{-} \times\{\xi=$ $0\}, B_{3}=\Omega \times\{\xi=0\} \times\{t=0\}$, and $B_{4}=\Omega \times\{\xi=0\} \times\{t=T\}$, let us consider $\delta>0$ and the set

$$
V_{S}^{\delta}=\left\{(x, \xi, t) \in \mathbb{R}^{d+2} \mid d_{S}(x, \xi, t)<3 \delta\right\}
$$

where $d_{S}(x, \xi, t)$ denote the distance of the point $(x, \xi, t)$ to the set $S$.

The construction is completed noting that for each $\delta>0$, there exists an open set $V_{c}^{\delta}$ such that the set

$$
\left(V_{0}, V_{T}, V_{\mathcal{D}^{+}}, V_{\mathcal{D}^{-}}, V_{\mathcal{D}^{0}}, V_{S}^{\delta}, V_{c}^{\delta}\right)
$$

defines a covering of $\mathbb{R}^{d+2}$ with $\bar{\Omega} \times \mathbb{R}_{+} \times\{t=0, T\} \cap V_{c}^{\delta}=\emptyset, \bar{\Omega} \times\{\xi=0\} \times[0, T] \cap V_{c}^{\delta}=\emptyset$.

A partition of unity is built on this covering denoted by

$$
\left(\omega_{0}^{\delta}, \omega_{T}^{\delta}, \omega_{\mathcal{D}^{+}}^{\delta}, \omega_{\mathcal{D}^{-}}^{\delta}, \omega_{\mathcal{D}^{0}}^{\delta}, \omega_{S}^{\delta}, \omega_{c}^{\delta}\right)
$$

From Hypothesis 2.1, the set $S$ is the union of a finite number of $\mathrm{C}^{1}$ manifolds of dimension at most $d$. Hence, following [2], the function $\omega_{S}^{\delta}$ can be chosen such that

$$
\left\{\begin{array}{l}
\omega_{S}^{\delta}(x, \xi, t)=1 \text { if } d_{S}(x, \xi, t)<\delta \\
\omega_{S}^{\delta}(x, \xi, t)=0 \text { if } d_{S}(x, \xi, t)>2 \delta \\
\text { Measure }\left(\operatorname{Supp}\left(\omega_{S}^{\delta}\right) \cap\{(x, \xi, t),|(x, \xi, t)| \leq R\}\right) \leq C(R) \delta^{2} \\
\sup \left(\left\|\partial_{\xi} \omega_{S}^{\delta}\right\|_{\mathrm{L}^{\infty}},\left\|\partial_{t} \omega_{S}^{\delta}\right\|_{\mathrm{L}^{\infty}}\right) \leq \frac{C}{\delta}
\end{array}\right.
$$


Let us now define the characteristic solution $v_{c}$ as follows

$$
v_{c}(x, \xi, t)=\left\{\begin{array}{lll}
l^{s}\left(x, \tau_{e}(x, \xi, t)\right) & \text { if } \quad\left(x, \tau_{e}(x, \xi, t)\right) \in \mathcal{D}_{T}^{+} \\
v^{0}(x, \zeta(0 ; x, \xi, t)) & \text { if } \tau_{e}(x, \xi, t)=0
\end{array}+\int_{\tau_{e}(x, \xi, t)}^{t} f(x, \zeta(s ; x, \xi, t), s) \mathrm{d} s,\right.
$$

formally defined on $\Omega \times[0,+\infty) \times[0, T]$. Then we have the following lemma:

Lemma 6.2. Hypothesis 2.1 is assumed to hold. Then the function $v_{c}$ is in $\mathrm{L}^{\infty}\left(\Omega \times \mathbb{R}_{+}^{*} \times(0, T)\right)$ and satisfies

$$
\left\|v_{c}\right\|_{\mathrm{L}^{\infty}\left(\Omega \times \mathbb{R}_{+}^{*} \times(0, T)\right)} \leq\left\|l^{s}\right\|_{\mathrm{L}^{\infty}\left(\mathcal{D}_{T}^{+}\right)}+\left\|v^{0}\right\|_{\mathrm{L}^{\infty}\left(\Omega \times \mathbb{R}_{+}^{*}\right)}+T\|f\|_{\mathrm{L}^{\infty}\left(\Omega \times \mathbb{R}_{+}^{*} \times(0, T)\right)} .
$$

Proof. From Hypothesis 2.1, the set

$$
\left\{(x, \xi=\zeta(s ; x, 0, t), s) \mid s \in\left[\tau_{e}(x, 0, t), \tau_{s}(x, 0, t)\right],(x, t) \in \partial \mathcal{D}_{T}^{+} \cap \Omega \times(0, T)\right\}
$$

has a vanishing measure for the Lebesgue measure $\mathrm{d} x \mathrm{~d} \xi \mathrm{d} s$ (see the following computation of the term $\mathcal{I}_{1}$ ). We deduce from Lemma 6.1 that the function $v_{c}$ is defined a.e. on $\Omega \times \mathbb{R}_{+}^{*} \times(0, T)$. Let $\varphi \in \mathrm{C}_{c}^{\infty}\left(\Omega \times \mathbb{R}_{+}^{*} \times(0, T)\right)$ be a test function, and consider the integral

$$
\begin{aligned}
& \int_{\Omega \times \mathbb{R}_{+}^{*} \times(0, T)} v_{c}(x, \xi, t) \varphi(x, \xi, t) \mathrm{d} x \mathrm{~d} \xi \mathrm{d} t=\mathcal{I}_{1}+\mathcal{I}_{2}+\mathcal{I}_{3} \text { with } \\
& \mathcal{I}_{1}=\int_{\left\{(x, \xi, t) \in \Omega \times \mathbb{R}_{+}^{*} \times(0, T) \mid\left(x, \tau_{e}(x, \xi, t)\right) \in \mathcal{D}_{T}^{+}\right\}} l^{s}\left(x, \tau_{e}(x, \xi, t)\right) \varphi(x, \xi, t) \mathrm{d} x \mathrm{~d} \xi \mathrm{d} t, \\
& \mathcal{I}_{2}=\int_{\left\{(x, \xi, t) \in \Omega \times \mathbb{R}_{+}^{*} \times(0, T) \mid \tau_{e}(x, \xi, t)=0\right\}} v^{0}(x, \zeta(0 ; x, \xi, t)) \varphi(x, \xi, t) \mathrm{d} x \mathrm{~d} \xi \mathrm{d} t, \\
& \mathcal{I}_{3}=\int_{\Omega \times \mathbb{R}_{+}^{*} \times(0, T)} \int_{\tau_{e}(x, \xi, t)}^{t} f(x, \zeta(s ; x, \xi, t), s) \varphi(x, \xi, t) \mathrm{d} s \mathrm{~d} x \mathrm{~d} \xi \mathrm{d} t .
\end{aligned}
$$

Using the change of variables $(x, \xi, t)=(y, \zeta(s ; y, 0, \tau), s)$, the first term rewrites

$$
\begin{aligned}
\mathcal{I}_{1} & =\int_{\mathcal{D}_{T}^{+}} \int_{\tau}^{\tau_{s}(y, 0, \tau)} l^{s}(y, \tau) \partial_{t} h(y, \tau) \varphi(y, \zeta(s ; y, 0, \tau), s) \mathrm{d} s \mathrm{~d} y \mathrm{~d} \tau \\
& \leq\left\|l^{s}\right\|_{L^{\infty}\left(\mathcal{D}_{T}^{+}\right)} \int_{\mathcal{D}_{T}^{+}} \int_{\tau}^{\tau_{s}(y, 0, \tau)} \partial_{t} h(y, \tau)|\varphi(y, \zeta(s ; y, 0, \tau), s)| \mathrm{d} s \mathrm{~d} y \mathrm{~d} \tau
\end{aligned}
$$

Back to the original variables, we obtain that $\mathcal{I}_{1}$ is bounded by $\left\|l^{s}\right\|_{\mathrm{L}^{\infty}\left(\mathcal{D}_{T}^{+}\right)}\|\varphi\|_{\mathrm{L}^{1}\left(\Omega \times \mathbb{R}_{+}^{*} \times(0, T)\right)}$. Using the change of variables $(x, \xi, t)=(y, \zeta(s ; y, \eta, 0), s)$, the second term rewrites

$$
\mathcal{I}_{2}=\int_{\Omega} \int_{\mathbb{R}_{+}} \int_{0}^{\tau_{s}(y, \eta, 0)} v^{0}(y, \eta) \varphi(y, \zeta(s ; y, \eta, 0), s) \mathrm{d} s \mathrm{~d} \eta \mathrm{d} y
$$

for which we obtain the bound $\mathcal{I}_{2} \leq\left\|v^{0}\right\|_{\mathrm{L}^{\infty}\left(\Omega \times \mathbb{R}_{+}^{*}\right)}\|\varphi\|_{\mathrm{L}^{1}\left(\Omega \times \mathbb{R}_{+}^{*} \times(0, T)\right)}$. Finally, using the change of variable $(x, \xi, t, s)=\left(y, \zeta\left(t^{\prime} ; y, \eta, s^{\prime}\right), t^{\prime}, s^{\prime}\right)$, we obtain similarly that the last term $\mathcal{I}_{3}$ is bounded by

$$
T\|f\|_{\mathrm{L}^{\infty}\left(\Omega \times \mathbb{R}_{+}^{*} \times(0, T)\right)}\|\varphi\|_{\mathrm{L}^{1}\left(\Omega \times \mathbb{R}_{+}^{*} \times(0, T)\right)}
$$

which ends the proof.

Lemma 6.3. Hypothesis 2.1 is assumed to hold, then $\mathcal{L} v_{c}=f$. 
Proof. Let $\mathcal{T} \in \mathrm{L}^{\infty}\left(\Omega \times \mathbb{R}_{+}^{*} \times(0, T)\right)$ be such that for all $\left(x_{0}, \xi_{0}, t_{0}\right) \in \Omega \times \mathbb{R}_{+}^{*} \times(0, T)$, there exists a neighbourhood $V$ of $\left(x_{0}, \xi_{0}, t_{0}\right)$ in $\Omega \times \mathbb{R}_{+}^{*} \times(0, T)$ and $s \in(0, T)$ such that $\zeta(s ; x, \xi, t)>0$ and $\mathcal{T}(x, \zeta(s ; x, \xi, t), s)=\mathcal{T}(x, \xi, t)$ for all $(x, \xi, t) \in V$. Then, let us show that $\mathcal{L} \mathcal{T}=0$ in $\mathcal{D}^{\prime}\left(\Omega \times \mathbb{R}_{+}^{*} \times(0, T)\right)$ and hence in $\mathrm{L}^{\infty}\left(\Omega \times \mathbb{R}_{+}^{*} \times(0, T)\right)$. Let $\varphi$ be a test function in $\mathrm{C}_{c}^{\infty}(V)$, then one has

$$
\int_{V} \mathcal{T}(x, \xi, t)(\mathcal{L} \varphi)(x, \xi, t) \mathrm{d} x \mathrm{~d} \xi \mathrm{d} t=\int_{V} \mathcal{T}(x, \zeta(s ; x, \xi, t), s)(\mathcal{L} \varphi)(x, \xi, t) \mathrm{d} x \mathrm{~d} \xi \mathrm{d} t .
$$

Let us consider the change of variables $\left(x^{\prime}, \xi^{\prime}, t^{\prime}\right)=(x, \zeta(s ; x, \xi, t), t)$, mapping $V^{\prime}$ to $V$, and the function $\phi$ such that $\phi\left(x^{\prime}, \xi^{\prime}, t^{\prime}\right)=\varphi(x, \xi, t)$ on $V^{\prime}$. One has

$$
\int_{V} \mathcal{T}(x, \xi, t)(\mathcal{L} \varphi)(x, \xi, t) \mathrm{d} x \mathrm{~d} \xi \mathrm{d} t=\int_{V^{\prime}} \mathcal{T}\left(x^{\prime}, \xi^{\prime}, s\right) \partial_{t^{\prime}} \phi\left(x^{\prime}, \xi^{\prime}, t^{\prime}\right) \mathrm{d} x^{\prime} \mathrm{d} \xi^{\prime} \mathrm{d} t^{\prime}=0
$$

To conclude that $\mathcal{L} \mathcal{T}=0$, it suffices to consider a test function $\psi \in \mathrm{C}_{c}^{\infty}\left(\Omega \times \mathbb{R}_{+}^{*} \times(0, T)\right)$, a finite covering of $\operatorname{Supp}(\psi)$ satisfying the above property, and a partition of unity on this covering.

The above property is clearly satisfied for the functions $\tau_{e}, \mathcal{T}_{1}$, and $\mathcal{T}_{2}$ with

$$
\mathcal{T}_{1}(x, \xi, t)=l^{s}\left(x, \tau_{e}(x, \xi, t)\right) \chi_{\left\{(x, \xi, t) \in \Omega \times \mathbb{R}_{+}^{*} \times(0, T) \mid\left(x, \tau_{e}(x, \xi, t)\right) \in \mathcal{D}_{T}^{+}\right\}}
$$

and

$$
\mathcal{T}_{2}(x, \xi, t)=v^{0}(x, \zeta(0 ; x, \xi, t)) \chi_{\left\{(x, \xi, t) \in \Omega \times \mathbb{R}_{+}^{*} \times(0, T) \mid \tau_{e}(x, \xi, t)=0\right\}}
$$

on $\Omega \times \mathbb{R}_{+}^{*} \times(0, T)$. We deduce that $\mathcal{L}\left(\tau_{e}\right)=\mathcal{L} \mathcal{T}_{1}=\mathcal{L} \mathcal{T}_{2}=0$, and also that $\mathcal{L} \mathcal{T}_{3}=f$ with $\mathcal{T}_{3}$ the function defined by $\mathcal{T}_{3}(x, \xi, t)=\int_{\tau_{e}(x, \xi, t)}^{t} f(x, \zeta(s ; x, \xi, t), s) \mathrm{d} s$, which finally proves that $\mathcal{L} v_{c}=\mathcal{L} \mathcal{T}_{1}+\mathcal{L} \mathcal{T}_{2}+\mathcal{L} \mathcal{T}_{3}=f$.

In order to prove the integration by part formula (4.3), we need the following lemma which is a direct application of the up-to-the boundary Friedrichs' lemma ([3], Cor. 3.2, p. 882).

Lemma 6.4. The function space $\mathrm{C}_{c}^{\infty}\left(\bar{\Omega} \times \mathbb{R}_{+} \times[0, T]\right)$ is dense in the Hilbert space

$$
W_{\mathcal{L}}\left(\Omega \times \mathbb{R}_{+}^{*} \times(0, T)\right)=\left\{v \in \mathrm{L}^{2}\left(\Omega \times \mathbb{R}_{+}^{*} \times(0, T)\right) \text { such that } \mathcal{L} v \in \mathrm{L}^{2}\left(\Omega \times \mathbb{R}_{+}^{*} \times(0, T)\right)\right\}
$$

endowed with the norm $\|v\|_{W_{\mathcal{L}}\left(\Omega \times \mathbb{R}_{+}^{*} \times(0, T)\right)}=\|v\|_{L^{2}\left(\Omega \times \mathbb{R}_{+}^{*} \times(0, T)\right)}+\|\mathcal{L} v\|_{L^{2}\left(\Omega \times \mathbb{R}_{+}^{*} \times(0, T)\right)}$.

Let $\mathbf{n}=\left(n_{x}, n_{\xi}, n_{t}\right)$ denote the unit vector normal to the boundary $\partial\left[\Omega \times \mathbb{R}_{+}^{*} \times(0, T)\right]$ outward to $\Omega \times$ $\mathbb{R}_{+}^{*} \times(0, T)$ and defined almost everywhere. It results from Lemma 6.4 that we can define by prolongation the continuous trace operator $\gamma_{\mathcal{L}}$ from $W_{\mathcal{L}}\left(\Omega \times \mathbb{R}_{+}^{*} \times(0, T)\right)$ to $L^{2}\left[\Omega ; \mathrm{H}^{-1 / 2}\left(\partial\left[\mathbb{R}_{+}^{*} \times(0, T)\right]\right)\right]$ such that for all $\varphi \in \mathrm{C}_{c}^{\infty}\left(\bar{\Omega} \times \mathbb{R}_{+} \times[0, T]\right), \gamma_{\mathcal{L}} \varphi=\left.\varphi\left(n_{t}+\partial_{t} h n_{\xi}\right)\right|_{\Omega \times \partial\left[\mathbb{R}_{+}^{*} \times(0, T)\right]}$, and satisfying

$$
\begin{gathered}
\int_{\Omega} \int_{\mathbb{R}_{+}} \int_{0}^{T} \phi(x, \xi, t) \mathcal{L} v(x, \xi, t) \mathrm{d} t \mathrm{~d} \xi \mathrm{d} x+\int_{\Omega} \int_{\mathbb{R}_{+}} \int_{0}^{T} v(x, \xi, t) \mathcal{L} \phi(x, \xi, t) \mathrm{d} t \mathrm{~d} \xi \mathrm{d} x \\
=\int_{\Omega} \int_{\partial\left[\mathbb{R}_{+}^{*} \times(0, T)\right]} \phi(x, \xi, t) \gamma_{\mathcal{L}} v(x, \xi, t) \mathrm{d} \sigma \mathrm{d} x,
\end{gathered}
$$

for all $\phi \in L^{2}\left[\Omega ; H^{1}\left(\mathbb{R}_{+}^{*} \times(0, T)\right)\right]$, where the last integral on $\Omega \times \partial\left[\mathbb{R}_{+}^{*} \times(0, T)\right]$ is taken in the sense of the duality product.

The next lemma states that (4.3) is satisfied for any function $v \in \mathrm{L}^{\infty}\left(\Omega \times \mathbb{R}_{+}^{*} \times(0, T)\right)$ such that $\mathcal{L} v \in$ $\mathrm{L}^{\infty}\left(\Omega \times \mathbb{R}_{+}^{*} \times(0, T)\right)$, which is the case in particular for $v=v_{c}$ from Lemmae 6.2 and 6.3 . 
Lemma 6.5. For any function $v \in \mathrm{L}^{\infty}\left(\Omega \times \mathbb{R}_{+}^{*} \times(0, T)\right)$ such that $\mathcal{L} v \in \mathrm{L}^{\infty}\left(\Omega \times \mathbb{R}_{+}^{*} \times(0, T)\right)$, the trace $\gamma_{\mathcal{L}} v$ belongs to $L^{\infty}\left(\Omega \times \partial\left[\mathbb{R}_{+}^{*} \times(0, T)\right]\right)$ and satisfies the estimate

$$
\left\|\gamma_{\mathcal{L}} v\right\|_{L^{\infty}\left(\Omega \times \partial\left[\mathbb{R}_{+}^{*} \times(0, T)\right]\right)} \leq C\left(\|v\|_{L^{\infty}\left(\Omega \times \mathbb{R}_{+}^{*} \times(0, T)\right)}+\|\mathcal{L} v\|_{L^{\infty}\left(\Omega \times \mathbb{R}_{+}^{*} \times(0, T)\right)}\right)
$$

with $C$ independent of $v$. This trace is denoted by $v_{c}(., 0,.) \partial_{t} h$ on $\xi=0, v_{c}(., ., 0)$ on $t=0$, and $v_{c}(., ., T)$ on $t=T$ and we have the integration by part formula

$$
\begin{gathered}
\int_{\Omega} \int_{\mathbb{R}_{+}} \int_{0}^{T}((\mathcal{L} \phi)(x, \xi, t) v(x, \xi, t)+(\mathcal{L} v)(x, \xi, t) \phi(x, \xi, t)) \mathrm{d} t \mathrm{~d} \xi \mathrm{d} x \\
+\int_{\Omega} \int_{\mathbb{R}_{+}}(v(x, \xi, 0) \phi(x, \xi, 0)-v(x, \xi, T) \phi(x, \xi, T)) \mathrm{d} \xi \mathrm{d} x \\
\quad+\int_{\Omega} \int_{0}^{T} \partial_{t} h(x, t) v(x, 0, t) \phi(x, 0, t) \mathrm{d} t \mathrm{~d} x=0 .
\end{gathered}
$$

for all $\phi \in L^{1}\left[\Omega ; W^{1,1}\left(\mathbb{R}_{+}^{*} \times(0, T)\right)\right]$.

Proof. Since $v$ and $\mathcal{L} v$ belong to $\mathrm{L}^{\infty}\left(\Omega \times \mathbb{R}_{+}^{*} \times(0, T)\right),(6.5)$ holds for all $\phi \in \mathrm{C}_{c}^{\infty}\left(\mathbb{R}^{d+2}\right)$.

It is known that $\mathrm{C}_{c}^{\infty}\left(\bar{\Omega} \times \mathbb{R}_{+} \times[0, T]\right)$ is dense in $L^{1}\left[\Omega ; W^{1,1}\left(\mathbb{R}_{+}^{*} \times(0, T)\right)\right]$, and that the trace operator from $L^{1}\left[\Omega ; W^{1,1}\left(\mathbb{R}_{+}^{*} \times(0, T)\right)\right]$ to $L^{1}\left(\Omega \times \partial\left[\mathbb{R}_{+}^{*} \times(0, T)\right]\right)$ is onto. It results that the set of traces of $\mathrm{C}_{c}^{\infty}\left(\bar{\Omega} \times \mathbb{R}_{+} \times[0, T]\right)$ on $\Omega \times \partial\left[\mathbb{R}_{+}^{*} \times(0, T)\right]$ defines a space denoted by $\mathrm{C}_{c}^{\infty}\left(\bar{\Omega} \times \partial\left[\mathbb{R}_{+}^{*} \times(0, T)\right]\right)$ which is dense in $L^{1}\left(\Omega \times \partial\left[\mathbb{R}_{+}^{*} \times(0, T)\right]\right)$.

For any function $\theta$ of $\mathrm{C}_{c}^{\infty}\left(\bar{\Omega} \times \partial\left[\mathbb{R}_{+}^{*} \times(0, T)\right]\right)$, one can built a function $\phi \in L^{2}\left[\Omega ; H^{1}\left(\mathbb{R}_{+}^{*} \times(0, T)\right)\right]$ compactly supported and such that

$$
\|\phi\|_{L^{1}\left[\Omega ; W^{1,1}\left(\mathbb{R}_{+}^{*} \times(0, T)\right)\right]} \leq C\|\theta\|_{L^{1}\left(\Omega \times \partial\left[\mathbb{R}_{+}^{*} \times(0, T)\right]\right)}
$$

with $\mathrm{C}$ independent of $\theta$. Hence we have

$$
\int_{\Omega} \int_{\partial\left[\mathbb{R}_{+}^{*} \times(0, T)\right]} \theta \gamma_{\mathcal{L}} v \mathrm{~d} \sigma \mathrm{d} x \leq C\left(\|v\|_{L^{\infty}\left(\Omega \times \mathbb{R}_{+}^{*} \times(0, T)\right)}+\|\mathcal{L} v\|_{L^{\infty}\left(\Omega \times \mathbb{R}_{+}^{*} \times(0, T)\right)}\right)\|\theta\|_{L^{1}\left(\Omega \times \partial\left[\mathbb{R}_{+}^{*} \times(0, T)\right]\right)}
$$

for all $\theta \in \mathrm{C}_{c}^{\infty}\left(\bar{\Omega} \times \partial\left[\mathbb{R}_{+}^{*} \times(0, T)\right]\right)$. By density, we conclude that $\gamma_{\mathcal{L}} v$ belongs to $L^{\infty}\left(\Omega \times \partial\left[\mathbb{R}_{+}^{*} \times(0, T)\right]\right)$. The integration by part formula results from (6.5) and the density of $\mathrm{C}_{c}^{\infty}\left(\bar{\Omega} \times \mathbb{R}_{+} \times[0, T]\right)$ in $L^{1}\left[\Omega ; W^{1,1}\left(\mathbb{R}_{+}^{*} \times\right.\right.$ $(0, T))]$.

We shall now use the partition of unity to prove that $v_{c}$ is a weak solution i.e. that $v_{c}(., 0,.) \partial_{t} h=l^{s}$ on $\mathcal{D}_{T}^{+}$ and $v_{c}(., ., 0)=v^{0}$.

Lemma 6.6. Hypothesis 2.1 is assumed to hold, then $v_{c}$ is a weak solution to (4.1).

Proof. Let us consider a function $\varphi$ in $\mathrm{C}_{c}^{\infty}\left(\mathbb{R}^{d+2}\right)$ such that $\varphi(., 0,)=$.0 on $\Omega \times(0, T) \backslash \mathcal{D}_{T}^{+}$and $\varphi(., ., T)=0$ on $\Omega \times \mathbb{R}_{+}^{*}$. By construction of the sets $V_{0}$ and $V_{\mathcal{D}^{+}}$, assuming smooth data $f, v^{0}$, and $l^{s}$, the function $v_{c}$ is smooth on the sets $V_{0} \cap \bar{\Omega} \times \mathbb{R}_{+} \times[0, T]$ and $V_{\mathcal{D}^{+}} \cap \bar{\Omega} \times \mathbb{R}_{+} \times[0, T]$. Hence one has

$$
\begin{gathered}
\int_{\Omega} \int_{\mathbb{R}_{+}} \int_{0}^{T}\left(\mathcal{L}\left(\varphi \omega_{0}^{\delta}\right)(x, \xi, t) v_{c}(x, \xi, t)+f(x, \xi, t)\left(\varphi \omega_{0}^{\delta}\right)(x, \xi, t)\right) \mathrm{d} t \mathrm{~d} \xi \mathrm{d} x \\
+\int_{\Omega} \int_{\mathbb{R}_{+}} v^{0}(x, \xi)\left(\varphi \omega_{0}^{\delta}\right)(x, \xi, 0) \mathrm{d} \xi \mathrm{d} x=0,
\end{gathered}
$$

and

$$
\begin{aligned}
\int_{\Omega} \int_{\mathbb{R}_{+}} \int_{0}^{T}( & \left.\mathcal{L}\left(\varphi \omega_{\mathcal{D}^{+}}^{\delta}\right)(x, \xi, t) v_{c}(x, \xi, t)+f(x, \xi, t)\left(\varphi \omega_{\mathcal{D}^{+}}^{\delta}\right)(x, \xi, t)\right) \mathrm{d} t \mathrm{~d} \xi \mathrm{d} x \\
& +\int_{\Omega} \int_{0}^{T} \partial_{t} h(x, t) l^{s}(x, t)\left(\varphi \omega_{\mathcal{D}^{+}}^{\delta}\right)(x, 0, t) \mathrm{d} t \mathrm{~d} x=0 .
\end{aligned}
$$


From Lemma $6.2, v_{c}$ is a linear continuous function of $f, v^{0}$, and $l^{s}$ in $\mathrm{L}^{\infty}$ norms. We deduce that (6.7) and (6.8) extend by continuity and density to data $f, v^{0}$, and $l^{s}$ in $\mathrm{L}^{\infty}$.

By definition of $\varphi$ and (6.6) one has

$$
\int_{\Omega} \int_{\mathbb{R}_{+}} \int_{0}^{T}\left(\mathcal{L}\left(\varphi\left(\omega_{\mathcal{D}^{-}}^{\delta}+\omega_{T}^{\delta}\right)\right)(x, \xi, t) v_{c}(x, \xi, t)+f \varphi\left(\omega_{\mathcal{D}^{-}}^{\delta}+\omega_{T}^{\delta}\right)(x, \xi, t)\right) \mathrm{d} t \mathrm{~d} \xi \mathrm{d} x=0 .
$$

The equality

$$
\int_{\Omega} \int_{\mathbb{R}_{+}} \int_{0}^{T}\left(\mathcal{L}\left(\varphi\left(\omega_{\mathcal{D}^{0}}^{\delta}+\omega_{c}^{\delta}\right)\right)(x, \xi, t) v_{c}(x, \xi, t)+f \varphi\left(\omega_{\mathcal{D}^{0}}^{\delta}+\omega_{c}^{\delta}\right)(x, \xi, t)\right) \mathrm{d} t \mathrm{~d} \xi \mathrm{d} x=0
$$

which clearly holds for any smooth function, readily extends by density from Lemma 6.4 to $v_{c}$ since the functions $v_{c}$ and $\mathcal{L} v_{c}$ are in $\mathrm{L}^{\infty}\left(\Omega \times \mathbb{R}_{+}^{*} \times(0, T)\right)$ and $\varphi$ is compactly supported.

Finally, from the properties (6.3) verified by the function $\omega_{S}^{\delta}$, one has

$$
\left|\int_{\Omega} \int_{\mathbb{R}_{+}} \int_{0}^{T}\left(\mathcal{L}\left(\varphi \omega_{S}^{\delta}\right)(x, \xi, t) v_{c}(x, \xi, t)+f(x, \xi, t)\left(\varphi \omega_{S}^{\delta}\right)(x, \xi, t)\right) \mathrm{d} t \mathrm{~d} \xi \mathrm{d} x\right| \leq C\left(\varphi, v_{c}\right) \delta
$$

where $C\left(\varphi, v_{c}\right)$ only depends on $\varphi$ and $v_{c}$. Passing to the limit $\delta \rightarrow 0$, we conclude that $v_{c}$ is a weak solution.

The following lemma states that $v_{c}$ is the unique weak solution to (4.1). The proof uses again the partition of unity.

Lemma 6.7. Hypothesis 2.1 is assumed to hold, then $v_{c}$ is the unique weak solution to (4.1).

Proof. Let $u$ be a weak solution of (4.1) with $f=v^{0}=l^{s}=0$. In the sense of distributions, it results that $\mathcal{L} u=0$ on $\Omega \times \mathbb{R}_{+}^{*} \times(0, T)$, and that the trace $\gamma_{\mathcal{L}} u\left(\right.$ in $L^{\infty}\left(\Omega \times \partial\left[\mathbb{R}_{+}^{*} \times(0, T)\right]\right)$ from Lemma 6.5) vanishes on $\mathcal{D}_{T}^{+} \cup \Omega \times \mathbb{R}_{+}^{*} \times\{t=0\}$. On the sets $V_{0} \cap \Omega \times \mathbb{R}_{+}^{*} \times[0, T)$, and $V_{\mathcal{D}^{+}} \cap \Omega \times \mathbb{R}_{+} \times(0, T)$, the equation $\mathcal{L} u=0$ can be integrated along the characteristics using the boundary conditions leading to $u=0$ on these sets. For any function $\psi$ in $\mathrm{C}_{c}^{\infty}\left(\mathbb{R}^{d+2}\right)$, let us consider the compactly supported function

$$
w(x, \xi, t)=\int_{\Omega} \int_{\mathbb{R}_{+}} \int_{0}^{T} \int_{\tau_{s}(x, \xi, t)}^{t} \psi(x, \zeta(s ; x, \xi, t), s) \mathrm{d} s \mathrm{~d} t \mathrm{~d} \xi \mathrm{d} x
$$

which is, from the previous results applied to the adjoint system, a weak solution to

$$
\left\{\begin{aligned}
-\mathcal{L} w=\psi & \text { on } \Omega \times \mathbb{R}_{+}^{*} \times(0, T) \\
\left.w\right|_{\xi=0}=0 & \text { on } \mathcal{D}_{T}^{-} \\
\left.w\right|_{t=T}=0 & \text { on } \Omega \times \mathbb{R}_{+}^{*}
\end{aligned}\right.
$$

Since $u=0$ on $\left(V_{0} \cup V_{\mathcal{D}^{+}}\right) \cap \Omega \times \mathbb{R}_{+} \times[0, T]$, one clearly has

$$
\int_{\Omega} \int_{\mathbb{R}_{+}} \int_{0}^{T} \mathcal{L}\left[u w\left(\omega_{\mathcal{D}^{+}}^{\delta}+\omega_{0}^{\delta}\right)\right](x, \xi, t) \mathrm{d} t \mathrm{~d} \xi \mathrm{d} x=0 .
$$

Let $\rho$ be a function in $\mathrm{C}_{c}^{\infty}\left(\mathbb{R}^{d+2}\right)$ such that $\rho=1$ on the compact support of $w$. Since $\rho u$ belongs to $W_{\mathcal{L}}\left(\Omega \times \mathbb{R}_{+}^{*} \times\right.$ $(0, T))$ and $w$ is smooth on $\left(V_{T} \cup V_{\mathcal{D}^{-}}\right) \cap \bar{\Omega} \times \mathbb{R}_{+} \times[0, T]$, equation (6.5) applied to $v=\rho u$ and $\phi=w\left(\omega_{\mathcal{D}^{-}}^{\delta}+\omega_{T}^{\delta}\right)$ 
yields that

$$
\int_{\Omega} \int_{\mathbb{R}_{+}} \int_{0}^{T} \mathcal{L}\left[u w\left(\omega_{\mathcal{D}^{-}}^{\delta}+\omega_{T}^{\delta}\right)\right](x, \xi, t) \mathrm{d} t \mathrm{~d} \xi \mathrm{d} x=0 .
$$

Also, since $(u w)$ belongs to $W_{\mathcal{L}}\left(\Omega \times \mathbb{R}_{+}^{*} \times(0, T)\right)$, by density of the smooth functions in $W_{\mathcal{L}}\left(\Omega \times \mathbb{R}_{+}^{*} \times(0, T)\right)$ we conclude that

$$
\int_{\Omega} \int_{\mathbb{R}_{+}} \int_{0}^{T} \mathcal{L}\left[u w\left(\omega_{\mathcal{D}^{0}}^{\delta}+\omega_{c}^{\delta}\right)\right](x, \xi, t) \mathrm{d} t \mathrm{~d} \xi \mathrm{d} x=0
$$

Finally, as above

$$
\left|\int_{\Omega} \int_{\mathbb{R}_{+}} \int_{0}^{T} \mathcal{L}\left[u w \omega_{S}^{\delta}\right](x, \xi, t) \mathrm{d} t \mathrm{~d} \xi \mathrm{d} x\right| \leq C(u, w) \delta,
$$

and passing to the limit $\delta \rightarrow 0$, we conclude that

$$
\int_{\Omega} \int_{\mathbb{R}_{+}} \int_{0}^{T} \mathcal{L}(u w)(x, \xi, t) \mathrm{d} t \mathrm{~d} \xi \mathrm{d} x=-\int_{\Omega} \int_{\mathbb{R}_{+}} \int_{0}^{T} \psi(x, \xi, t) u(x, \xi, t) \mathrm{d} t \mathrm{~d} \xi \mathrm{d} x=0
$$

for all $\psi \in \mathrm{C}_{c}^{\infty}\left(\mathbb{R}^{d+2}\right)$, which ends the proof.

\section{Proof of the integration by part formula (4.5)}

From Lemma 6.7, the solutions $v$ and $w$ of the direct and adjoint equations (4.1) and (4.4) are defined by their characteristic solutions, and $w$ is compactly supported on $\bar{\Omega} \times \mathbb{R}_{+} \times[0, T]$. From Lemmae 6.2 and 6.5, the functions $v$ and $w$ and their traces depend continuously on the data $f, v^{0}, l^{s}, r, q^{s}$, and $w^{T}$ in $\mathrm{L}^{\infty}$ norms so that it suffices to prove the integration by part formula (4.5) assuming smooth data.

Let $\rho$ be a function in $\mathrm{C}_{c}^{\infty}\left(\mathbb{R}^{d+2}\right)$ such that $\rho=1$ on the compact support of $w$. On the sets $V_{0} \cap \bar{\Omega} \times \mathbb{R}_{+} \times[0, T]$ and $V_{\mathcal{D}^{+}} \cap \bar{\Omega} \times \mathbb{R}_{+} \times[0, T]$, the solution $v$ is smooth and we can apply the integration by part formula (4.3) to $w$ and $\varphi=v\left(\omega_{\mathcal{D}^{+}}^{\delta}+\omega_{0}^{\delta}\right) \rho$. Similarly, on the sets $V_{T} \cap \bar{\Omega} \times \mathbb{R}_{+} \times[0, T]$ and $V_{\mathcal{D}^{-}} \cap \bar{\Omega} \times \mathbb{R}_{+} \times[0, T]$, the solution $w$ is smooth and we can apply the integration by part formula (4.3) to $v$ and $\varphi=w\left(\omega_{\mathcal{D}^{-}}^{\delta}+\omega_{T}^{\delta}\right)$. Since $(v w)$ belongs to the function space $W_{\mathcal{L}}\left(\Omega \times \mathbb{R}_{+}^{*} \times(0, T)\right)$, it results from the density Lemma 6.4 that

$$
\int_{\Omega} \int_{\mathbb{R}_{+}} \int_{0}^{T} \mathcal{L}\left[v w\left(\omega_{\mathcal{D}^{0}}^{\delta}+\omega_{c}^{\delta}\right)\right](x, \xi, t) \mathrm{d} t \mathrm{~d} \xi \mathrm{d} x=0
$$

We conclude the proof using the estimate

$$
\left|\int_{\Omega} \int_{\mathbb{R}_{+}} \int_{0}^{T} \mathcal{L}\left[v w \omega_{S}^{\delta}\right](x, \xi, t) \mathrm{d} t \mathrm{~d} \xi \mathrm{d} x\right| \leq C(v, w) \delta,
$$

and passing to the limit $\delta \rightarrow 0$.

\section{Proof of Lemma 4.3}

We shall need the following lemma which is proved using the up-to-the boundary version of Friedrich's lemma ([3], Cor. 3.2, p. 882).

Lemma 7.1. The function space $\mathrm{C}^{\infty}(\bar{\Omega} \times[0, T])$ is dense in the Hilbert space

$$
W_{h}(\Omega \times(0, T))=\left\{v \in \mathrm{L}^{2}(\Omega \times(0, T)) \text { such that } \nabla h \cdot \nabla v \in \mathrm{L}^{2}(\Omega \times(0, T))\right\}
$$

endowed with the norm $\|v\|_{W_{h}(\Omega \times(0, T))}=\|v\|_{\mathrm{L}^{2}(\Omega \times(0, T))}+\|\nabla h \cdot \nabla v\|_{\mathrm{L}^{2}(\Omega \times(0, T))}$. 
It results from Lemma 7.1 that we can define by prolongation the continuous trace operator $\gamma_{h}$ from $W_{h}(\Omega \times$ $(0, T))$ to $L^{2}\left[(0, T) ; \mathrm{H}^{-1 / 2}(\partial \Omega)\right]$ such that for all $\varphi \in \mathrm{C}^{\infty}(\bar{\Omega} \times[0, T]), \gamma_{h} \varphi=\left.\varphi \nabla h \cdot n_{x}\right|_{\partial \Omega \times(0, T)}$, and

$$
\int_{\Omega} \int_{0}^{T} \operatorname{div}(\phi v \nabla h) \mathrm{d} t \mathrm{~d} x=\int_{0}^{T} \int_{\partial \Omega} \phi \gamma_{h} v \mathrm{~d} t \mathrm{~d} \sigma
$$

for all $\phi \in L^{2}\left[(0, T) ; H^{1}(\Omega)\right]$, where the last integral on the boundary $\partial \Omega \times(0, T)$ is taken in the sense of the duality product.

As in the previous section, one can prove the following lemma.

Lemma 7.2. For any function $v \in L^{\infty}(\Omega \times(0, T))$ such that $\nabla h \cdot \nabla v \in L^{\infty}(\Omega \times(0, T))$, the trace $\gamma_{h} v$ is in $L^{\infty}(\partial \Omega \times(0, T))$ and one has

$$
\int_{\Omega} \int_{0}^{T} \operatorname{div}(\phi v \nabla h) \mathrm{d} t \mathrm{~d} x=\int_{0}^{T} \int_{\partial \Omega} \phi \gamma_{h} v \mathrm{~d} t \mathrm{~d} \sigma,
$$

for all $\phi \in L^{1}\left[(0, T) ; W^{1,1}(\Omega)\right]$.

From (4.7) and Lemma 7.2 it results that the trace $\gamma_{h} d_{i}^{s}$ is in $L^{\infty}(\partial \Omega \times(0, T))$. From (4.6) and Lemma 7.2, we deduce that $\gamma_{h} d_{i}^{s}$ vanishes on $\Sigma_{T}^{+}$. The same remarks hold for the trace $\gamma_{h} q_{i}^{s}$ on $\Sigma_{T}^{-}$. Since $\nabla h \cdot n_{x}>0$ on $\Sigma_{T}^{+}$and $\nabla h \cdot n_{x}<0$ on $\Sigma_{T}^{-}$, it results that $d_{i}^{s}$ has a vanishing trace on $\Sigma_{T}^{+}$and $q_{i}^{s}$ a vanishing trace on $\Sigma_{T}^{-}$.

From equations (4.10) and (4.7), the functions $q_{i}^{s} d_{i}^{s}$ and $\nabla h \cdot \nabla\left(q_{i}^{s} d_{i}^{s}\right)$ belong to $L^{\infty}(\Omega \times(0, T))$. Hence the trace $\gamma_{h}\left(q_{i}^{s} d_{i}^{s}\right)$ is in $L^{\infty}(\partial \Omega \times(0, T))$. To prove Lemma 4.3 , we need to show that $\gamma_{h}\left(q_{i}^{s} d_{i}^{s}\right)=0$. The proof will proceed as in the previous section using a covering of the domain $\bar{\Omega} \times[0, T]$ built from the trajectories of the vector field $\nabla h$ and a partition of unity. To work away from the set $Z=\partial \Sigma_{T}^{+} \cup \partial \Sigma_{T}^{-} \cup \partial \Omega \times\{t=0, T\}$, the trick from [2] and Hypothesis 2.1 will again be used.

Let $\omega$ be an open neighbourhood of $\bar{\Omega}$, and $\bar{h}$ a $\mathrm{C}^{2}$ extension of $h$ on $\omega \times[0, T]$. Let us define for all $(x, t) \in \omega \times(0, T)$, the trajectories $X(\tau ; x, t)$ of the vector field $\nabla \bar{h}$ as the maximal solutions in $\omega$ of

$$
\left\{\begin{aligned}
\frac{\partial X}{\partial \tau}(\tau ; x, t) & =\nabla \bar{h}(X(\tau ; x, t), t), \\
X(0 ; x, t) & =x .
\end{aligned}\right.
$$

Since $\nabla h \cdot n_{x}>0$ on $\Sigma_{T}^{+}$, for all $a \in \Sigma_{T}^{+}$, there exists an open neighbourhood $v_{a}$ of $a$ in $\Sigma_{T}^{+}$and $1>\epsilon_{a}>0$ such that the set

satisfies

$$
\mathcal{V}_{a}=\left\{(X(\tau ; x, t), t) \mid(x, t) \in v_{a}, \tau \in\left(-\epsilon_{a}, \epsilon_{a}\right)\right\}
$$

(i) $\mathcal{V}_{a}$ is an open neighbourhood of $a$ in $\omega \times(0, T)$;

(ii) the mapping $\left(x^{\prime}=X(\tau ; x, t), t^{\prime}=t\right)$ defines a $\mathrm{C}^{1}$ diffeomorphism from $v_{a} \times\left(-\epsilon_{a}, \epsilon_{a}\right)$ to $\mathcal{V}_{a}$. It results that the set

$$
W_{+}=\bigcup_{a \in \Sigma_{T}^{+}} \mathcal{V}_{a}
$$

is an open neighbourhood of $\Sigma_{T}^{+}$in $\omega \times(0, T)$. Let us denote by $W_{-}$the open neighbourhood of $\Sigma_{T}^{-}$in $\omega \times(0, T)$ built similarly.

Let $\Sigma_{T}^{0}$ denote the interior of the set $\{(x, t) \in \partial \Omega \times(0, T) \mid g(x, t)=0\}$ in $\partial \Omega \times(0, T)$. From the regularity of the boundary of $\Omega$, we can define a neighbourhood $W_{0}$ of $\Sigma_{T}^{0}$ in $\omega \times(0, T)$ such that $W_{0} \cap\left(\bar{\Sigma}_{T}^{+} \cup \bar{\Sigma}_{T}^{-}\right)=\emptyset$. 
For all $(x, t) \in \mathbb{R}^{d+1}$, let $d_{Z}(x, t)$ denote the distance of the point $(x, t)$ to the set $Z=\partial \Sigma_{T}^{+} \cup \partial \Sigma_{T}^{-} \cup \partial \Omega \times\{t=$ $0, T\}$, and let $W_{Z}^{\delta}$ be the set

$$
W_{Z}^{\delta}=\left\{(x, t) \in \mathbb{R}^{d+1} \mid d_{Z}(x, t)<3 \delta\right\} .
$$

There exists an open set $W_{c}^{\delta}$ such that the set

$$
\left(W_{+}, W_{-}, W_{0}, W_{Z}^{\delta}, W_{c}^{\delta}\right)
$$

defines a covering of $\mathbb{R}^{d+1}$ with $\partial \Omega \times[0, T] \cap W_{c}^{\delta}=\emptyset$. A partition of unity is built on this covering denoted by

$$
\left(\theta_{+}^{\delta}, \theta_{-}^{\delta}, \theta_{0}^{\delta}, \theta_{Z}^{\delta}, \theta_{c}^{\delta}\right)
$$

From Hypothesis 2.1, the set $Z$ is the union of a finite number of manifolds of dimension at most $d-1$. Hence, following [2], the function $\theta_{Z}^{\delta}$ can be chosen such that

$$
\left\{\begin{array}{l}
\theta_{Z}^{\delta}(x, t)=1 \text { if } d_{Z}(x, t)<\delta \\
\theta_{Z}^{\delta}(x, t)=0 \text { if } d_{Z}(x, t)>2 \delta \\
\text { Measure }\left(\operatorname{Supp}\left(\theta_{Z}^{\delta}\right) \cap\{(x, t),|(x, t)| \leq R\}\right) \leq C(R) \delta^{2}, \\
\left\|\left|\nabla \theta_{Z}^{\delta}\right|\right\|_{L^{\infty}} \leq \frac{C}{\delta} .
\end{array}\right.
$$

Let us consider the change of variables $\left(x=X\left(\tau ; x^{\prime}, t^{\prime}\right), t=t^{\prime}\right)$ from $\bigcup_{a \in \Sigma_{T}^{+}} v_{a} \times\left(-\epsilon_{a}, \epsilon_{a}\right)$ to $W_{+}$, and let us denote by $\bar{d}_{i}^{s}, \bar{\beta}$ the functions such that $\bar{d}_{i}^{s}\left(x^{\prime}, t^{\prime}, \tau\right)=d_{i}^{s}\left(X\left(\tau ; x^{\prime}, t^{\prime}\right), t^{\prime}\right)$, and $\bar{\beta}\left(x^{\prime}, t^{\prime}, \tau\right)=\beta\left(X\left(\tau ; x^{\prime}, t^{\prime}\right), t^{\prime}\right)$ with $\beta=\left(\left.v_{i}\right|_{\xi=0}-d_{i}^{s}\right) \partial_{t} h$. Then, the function $\bar{d}_{i}^{s}$ satisfies the equation

$$
\left\{\begin{aligned}
\frac{\partial}{\partial \tau} \bar{d}_{i}^{s}\left(x^{\prime}, t^{\prime}, \tau\right) & =\bar{\beta}\left(x^{\prime}, t^{\prime}, \tau\right), \\
\bar{d}_{i}^{s}\left(x^{\prime}, t^{\prime}, 0\right) & =0
\end{aligned}\right.
$$

which yields

$$
\bar{d}_{i}^{s}\left(x^{\prime}, t^{\prime}, \tau\right)=\int_{0}^{\tau} \bar{\beta}\left(x^{\prime}, t^{\prime}, s\right) \mathrm{d} s,
$$

and

$$
\int_{W_{+}} d_{i}^{s}(x, t) \phi(x, t) \mathrm{d} x \mathrm{~d} t \leq\|\beta\|_{\mathrm{L}^{\infty}\left(W_{+} \cap \Omega \times(0, T)\right)}\|\phi\|_{\mathrm{L}^{1}\left(W_{+}\right)}
$$

for all $\phi \in \mathrm{C}_{c}^{\infty}\left(W_{+}\right)$.

Let us assume for an instant that $\beta$ is in $\mathrm{C}_{c}^{\infty}(\Omega \times(0, T))$. In that case, it results from (7.5) that the function $d_{i}^{s}$ is smooth on $W_{+} \cap \bar{\Omega} \times(0, T)$, so that we can apply (7.1) to $\phi=\theta_{+}^{\delta} d_{i}^{s}$ and $v=q_{i}^{s}$ :

$$
\int_{\Omega} \int_{0}^{T} \operatorname{div}\left(\theta_{+}^{\delta} d_{i}^{s} q_{i}^{s} \nabla h\right) \mathrm{d} t \mathrm{~d} x=0
$$

From (7.6), the equality (7.7) still holds by density and continuity for $\beta \in \mathrm{L}^{\infty}(\Omega \times(0, T))$ and hence for the true function $d_{i}^{s}$.

The same arguments applied to $q_{i}^{s}$ and $W_{-}$yield that

$$
\int_{\Omega} \int_{0}^{T} \operatorname{div}\left(\theta_{-}^{\delta} d_{i}^{s} q_{i}^{s} \nabla h\right) \mathrm{d} t \mathrm{~d} x=0
$$


Since the function $q_{i}^{s} d_{i}^{s}$ belongs to $W_{h}(\Omega \times(0, T))$, it results by density from Lemma 7.1 that

$$
\int_{\Omega} \int_{0}^{T} \operatorname{div}\left[\left(\theta_{0}^{\delta}+\theta_{c}^{\delta}\right) d_{i}^{s} q_{i}^{s} \nabla h\right] \mathrm{d} t \mathrm{~d} x=0 .
$$

Finally, from the properties (7.4) verified by the function $\theta_{Z}^{\delta}$, it is clear that

$$
\left|\int_{\Omega} \int_{0}^{T} \operatorname{div}\left(\theta_{Z}^{\delta} d_{i}^{s} q_{i}^{s} \nabla h\right) \mathrm{d} t \mathrm{~d} x\right| \leq C\left(h, d_{i}^{s}, q_{i}^{s}\right) \delta .
$$

Passing to the limit $\delta \rightarrow 0$ in equations (7.7)-(7.10) concludes the proof of Lemma 4.3.

Acknowledgements. The authors would like to thank Professors Thierry Gallouët from the Université de Provence and Robert Eymard from the Université de Marne la Vallée for fruitful discussions and comments during the elaboration of this work.

\section{REFERENCES}

[1] R.S. Anderson and N.F. Humphrey, Interaction of Weathering and Transport Processes in the Evolution of Arid Landscapes, in Quantitative Dynamics Stratigraphy, T.A. Cross Ed., Prentice Hall (1989) 349-361.

[2] C. Bardos, Problèmes aux limites pour les équations aux dérivées partielles du premier ordre à coefficients réels ; théorèmes d'approximation ; application à l'équation de transport. Ann. Sci. École Norm. Sup. 3 (1971) 185-233.

[3] A. Blouza, H. Le Dret, An up-to-the boundary version of Friedrichs' lemma and applications to the linear Koiter shell model. SIAM J. Math. Anal. 33 (2001) 877-895.

[4] R. Eymard, T. Gallouët, V. Gervais and R. Masson, Convergence of a numerical scheme for stratigraphic modeling. SIAM J. Numer. Anal. submitted.

[5] R. Eymard, T. Gallouët, D. Granjeon, R. Masson and Q.H. Tran, Multi-lithology stratigraphic model under maximum erosion rate constraint. Int. J. Numer. Meth. Eng. 60 (2004) 527-548.

[6] P.B. Flemings and T.E. Jordan, A synthetic stratigraphic model of foreland basin development. J. Geophys. Res. 94 (1989) 3851-3866.

[7] E. Godlewski and P.A. Raviart, Numerical Approximation of Hyperbolic Systems of Conservation Laws. Springer (1996).

[8] D. Granjeon, Modélisation Stratigraphique Déterministe: Conception et Applications d'un Modèle Diffusif 3D Multilithologique. Ph.D. Thesis, Géosciences Rennes, Rennes, France (1997).

[9] D. Granjeon and P. Joseph, Concepts and applications of a 3D multiple lithology, diffusive model in stratigraphic modeling, in J.W. Harbaugh et al. Eds., Numerical Experiments in Stratigraphy, SEPM Sp. Publ. 62 (1999).

[10] P.M. Kenyon and D.L. Turcotte, Morphology of a delta prograding by bulk sediment transport, Geological Society of America Bulletin 96 (1985) 1457-1465.

[11] O. Ladyzenskaja, V. Solonnikov and N. Ural'ceva, Linear and quasilinear equations of parabolic type. Transl. Math. Monogr. 23 (1968).

[12] J.C. Rivenaes, Application of a dual lithology, depth-dependent diffusion equation in stratigraphic simulation. Basin Research 4 (1992) 133-146.

[13] J.C. Rivenaes, Impact of sediment transport efficiency on large-scale sequence architecture: results from stratigraphic computer simulation. Basin Research 9 (1997) 91-105.

[14] D.M. Tetzlaff and J.W. Harbaugh, Simulating Clastic Sedimentation. Van Norstrand Reinhold, New York (1989).

[15] G.E. Tucker and R.L. Slingerland, Erosional dynamics, flexural isostasy, and long-lived escarpments: A numerical modeling study. J. Geophys. Res. 99 (1994) 229-243. 